\title{
Dispersion of finite-size particles probing inhomogeneous and anisotropic turbulence
}

Article

Accepted Version

Creative Commons: Attribution-Noncommercial-No Derivative Works 4.0

Mériaux, C. A., Teixeira, M. A. C., Monaghan, J. J., Cohen, R. and Cleary, P. (2020) Dispersion of finite-size particles probing inhomogeneous and anisotropic turbulence. European Journal of Mechanics \& Fluids - B/Fluids, 84. pp. 93-109. ISSN 09977546 doi: https://doi.org/10.1016/j.euromechflu.2020.05.015 Available at https://centaur.reading.ac.uk/90962/

It is advisable to refer to the publisher's version if you intend to cite from the work. See Guidance on citing.

To link to this article DOI: http://dx.doi.org/10.1016/j.euromechflu.2020.05.015

Publisher: Elsevier

All outputs in CentAUR are protected by Intellectual Property Rights law, including copyright law. Copyright and IPR is retained by the creators or other copyright holders. Terms and conditions for use of this material are defined in the End User Agreement.

www.reading.ac.uk/centaur 
Central Archive at the University of Reading

Reading's research outputs online 


\title{
Dispersion of finite-size particles probing inhomogeneous and anisotropic turbulence
}

\author{
C.A. Mériaux ${ }^{a}, *$, Miguel A. C. Teixeira ${ }^{b}$, Joe J. Monaghan ${ }^{c}$, Raymond \\ Cohen $^{\mathrm{d}}$, Paul Cleary ${ }^{\mathrm{d}}$ \\ ${ }^{a}$ School of Earth, Atmosphere and Environment, Monash University, Clayton, Australia \\ ${ }^{b}$ Department of Meteorology, University of Reading, Reading, UK \\ ${ }^{c}$ School of Physics and Astronomy, Monash University, Clayton, Australia \\ ${ }^{d}$ CSIRO Data61, Commonwealth Scientific and Industrial Research Organisation, \\ Clayton, Australia
}

\begin{abstract}
A series of 8 laboratory experiments was used to investigate the dynamics of a few almost neutrally-buoyant finite-size particles in the entire volume of a rectangular tank open to air and filled with water. Stirring was achieved by a cylinder executing a two-dimensional periodic Lissajoux figure. The rate and direction of stirring by the cylinder was varied. The particle motions were analyzed using a tracking method developed for the experimental design. The Reynolds number associated with the large-scale stirring motion was in a turbulent range of $[5,693-11,649]$ across all experiments. The absence of stirring in the direction of the cylinder axis, the constant interference of the cylinder with the eddies and the presence of walls and the free-surface resulted in a flow that was both inhomogeneous and anisotropic as recorded by the particle motion. Despite these unusual conditions, the single-particle dispersion across all experiments could be seen to follow a ballistic regime until
\end{abstract}

\footnotetext{
${ }^{*}$ Corresponding author

Email address: catherine.meriaux@monash.edu (C.A. Mériaux)
} 
about two-fifths of the particle Lagrangian velocity auto-correlation time $T_{L}$. It was followed by a brief diffusive regime between $T_{L}$ and $2.5 T_{L}$, after which the presence of the boundaries prevented further dispersion. Such evolution is consistent with classic predictions for fluid tracer dispersion in homogeneous and isotropic turbulence. Particle-pair dispersion was more complex. Both the fixed time-averaged and length-scale-dependent particle-pair dispersion rates averaged across pairs showed the ballistic dispersion regime, whereas the subsequent diffusive regime was better borne out by the length-scaledependent particle-pair dispersion. A super-diffusive Richardson regime was not unmistakably detected. Substantial variability was however found across the different pairs of particles, which was linked to differences in the decorrelation time of the velocity difference as a result of the inhomogeneity of the turbulence. For short initial separations, some particle pairs had a better separation of the time scales delimiting the ballistic and diffusive regimes and showed hints of a brief Richardson regime.

Keywords: Turbulence, Dispersion, Particle mixing, Experimental modelling

\section{1. Introduction}

2 In many contexts, from natural systems to industrial processing, the 3 transport, dispersion or mixing of particulate matter in turbulent flows comes 4 into play. In the oceans, understanding how the wind mixes an ever increas5 ing number of floating plastic fragments down into the water is at the heart 
of estimating how much plastic waste exists in our oceans. In industry, the transformation of ingredients fed to a vessel and stirred by the motion of an impeller is determined by judiciously choosing an impeller and its motion that will create a homogeneous end-product so that it can pass its primary quality control. In realistic contexts, turbulence is prone to be inhomogeneous and anisotropic.

Yet, although numerous applications have motivated intense research on these topics, theoretical, numerical or experimental studies have mostly focused on the behaviour of fluid parcels within turbulent flows (e.g. Toschi and Bodenschatz, 2009; Salazar and Collins, 2009; Balachandar and Eaton, 2010). Theoretically, Kolmogorov (1941a,b) showed that, in three-dimensional (3D) homogeneous and isotropic turbulence, energy cascades from the larger scales, where energy is injected, down to a length scale $\eta$, at which dissipation by molecular viscosity becomes important. In the Eulerian reference frame (i.e. in terms of variables defined at points fixed in space), the energy spectrum $E(k)$ as a function of wavenumber $k$ follows $E(k) \sim \varepsilon^{2 / 3} k^{-5 / 3}$, where $\varepsilon$ is the energy dissipation rate, in the inertial range, which lies in between the production and dissipation scales, $k_{f}<k<k_{\eta}$, where $k_{f}$ is the forcing wavenumber and $k_{\eta} \sim 1 / \eta$ is the dissipation wavenumber. In the same range, the equivalent to the $-5 / 3$ law can be expressed in physical space by velocity structure functions of order 2 , satisfying $C_{2}(l)=\Lambda C_{K} \varepsilon^{2 / 3} l^{2 / 3}$, where $l$ is the spatial separation, $C_{K}$ is a universal constant found to be equal to 2.01 for homogeneous and isotropic turbulence and $\Lambda$ is a constant equal to 1 in the case of a longitudinal structure function and $4 / 3$ in the case of a transverse structure function (Sreenivasan, 1995). For $k>k_{\eta}$, viscosity 
becomes important, and $E(k)$ rapidly decays. In the Lagrangian reference frame (i.e. in terms of time-dependent variables following particles originating at position $\boldsymbol{y}$ and with velocity $\boldsymbol{U}(\boldsymbol{x}(\boldsymbol{y}, t), t))$, properties of homogeneous and isotropic turbulence are characterized by the velocity structure functions $D(\tau)$ representing the variance of temporal increments of any velocity component $U, D(\tau)=\langle\delta U(\tau) \delta U(\tau)\rangle$, where $\delta U(\tau)=U(t+\tau)-U(t)$, and the velocity frequency spectrum $E(\omega)$, defined as the Fourier cosine transform of the velocity autocovariance $R(\tau)=\langle U(t) U(t+\tau)\rangle$. In the inertial range, $D(\tau)$ is predicted to scale as $D(\tau)=C_{0} \varepsilon \tau$ and $E(\omega)$ as $E(\omega)=\left(C_{0} / \pi\right) \varepsilon \omega^{-2}$, and the constant $C_{0}$ has been found to equal 5 (Monin and Yaglom, 2013; Ouellette et al., 2006).

When particles are neutrally buoyant and small compared to the Kolmogorov dissipative length scale $\eta$, they behave as tracers of the fluid motion by passively following the flow. The upper size limit $d_{p}$ for tracer behaviour was determined to be $d_{p}=5 \eta$ (e.g. Qureshi et al., 2007; Volk et al., 2011). Dispersion of tracer-like particles in homogeneous and isotropic 3D turbulence differentiates single-particle dispersion, which is defined by the meansquare displacement of a particle from its initial position, from particle-pair dispersion or relative dispersion, which involves the mean-square separation of a pair of particles. In the dispersion of a single particle, also called Taylor dispersion, the mean-square displacement varies as $t^{2}$ for short times (ballistic regime) and is proportional to $t$ in a long-time diffusion limit (Taylor, 1922; Einstein, 1956). The particle-pair or relative dispersion, however, has been described by three regimes (Batchelor, 1950; Richardson, 1926; Csanady, 1973; Bourgoin, 2015). In the inertial regime, where the initial separation 
between two particles, $|\boldsymbol{S}|=S_{0}$, is greater than $\eta$, a ballistic regime is expected, for which $\left\langle\left(|\boldsymbol{S}|-S_{0}\right)^{2}\right\rangle \propto t^{2}$ if $t \ll t_{0}=\left(S_{0}^{2} / \varepsilon\right)^{1 / 3}$. The time $t_{0}$ is identified as the time for which the two fluid elements "recall" their initial relative velocity when moving in an eddy of size $S_{0}$. When $t_{0} \ll t \ll T_{L}$, where $T_{L}$ is the Lagrangian velocity auto-correlation time, an intermediate super-diffusive regime, also named the Richardson regime, is expected, for which $\left(\left\langle|\boldsymbol{S}|-S_{0}\right)^{2}\right\rangle \propto t^{3}$. Physically, this is caused by the fact that the scale of the eddies contributing to relative dispersion, which in this phase lies in the inertial range, is proportional to the separation between the dispersing particles. Finally, when $t \gg T_{L}$, i.e. when the particle separation equals or exceeds the scale of the dominant, energy-containing eddies in the turbulence, the particles are expected to separate diffusively as $\left\langle\left(|\boldsymbol{S}|-S_{0}\right)^{2} \propto t\right.$. An alternative to the previous fixed time-averaged indicators of relative dispersion is a length-scale-dependent dispersion rate, which is defined through the finitescale Lyapunov exponent (FSLE). Given the spatial separation $\delta$ between two particle trajectories and the mean time $\langle\tau(\delta)\rangle$ that $\delta$ takes to be amplified by a factor $\rho$, then the (Lagrangian) FSLE is defined as $\lambda(\delta)=\ln \rho /\langle\tau(\delta)\rangle$. Dimensional arguments further establish that if $\left\langle|\boldsymbol{S}|^{2}\right\rangle \propto t^{2 / \zeta}$, then $\lambda(\delta) \propto \delta^{-\zeta}$ (Aurell et al., 1996; Boffetta et al., 2000). Boffetta and Sokolov (2002) showed that the advantage of averaging at a fixed scale separation, as opposed to at a fixed time, is that it removes crossover effects since all sampled particle pairs belong to the same scales and as a result they allow a better identification of the super-diffusive Richardson regime.

The experimental study of particle motion in turbulence has developed substantially in the last decade with the use of new optical (e.g. La Porta 
et al., 2001) and acoustic (e.g. Mordant et al., 2004) tracking techniques. The synchronization of multiple fast cameras or ultrasonic/laser Doppler velocimetry allows fully resolving the 3D particle trajectories in turbulent flows, but the measurements are limited to time intervals of a few Kolmogorov times. In many experiments, the von Kármán apparatus is used (e.g. Zandbergen and Dijkstra, 1987; Mordant et al., 2003; Gibert et al., 2010). This is a closed flow chamber filled with a carrier fluid and consisting of twocounter rotating disks generating the turbulence. Properties of turbulence are inferred from hot anemometry or tracer-like particles. The observation volume is commonly limited and selected relatively far from the disks to avoid anisotropy and inhomogeneity in the turbulence. In such ideal turbulent conditions, both laboratory experiments and numerical simulations have confirmed the theoretical predictions on the dispersion of tracers (Bourgoin, 2015; Xia et al., 2019; Boffetta and Sokolov, 2002; Biferale et al., 2008; Bitane et al., 2012). In particular, these studies have shown that observation of the Richardson regime requires a significant scale separation between the different lengths, $\eta, S_{0}, L_{i}$, where $L_{i}$ is the integral length scale, a statistically characteristic length related to the largest energy-containing eddies in the turbulence $\left(L_{i} \propto 1 / k_{f}\right)$.

Otherwise, experimental studies (Zimmermann et al., 2011; Fiabane et al., 2012; Qureshi et al., 2007; Bourgoin et al., 2011) have typically investigated the behaviour of particles in a size and density range of $d_{p} \sim[5-30] \eta$ and $\rho_{p}=[1-70] \rho_{a}$, where $\rho_{a}$ is the density of the ambient fluid, respectively. Qureshi et al. (2007) and Bourgoin et al. (2011) especially showed that the inertia of finite-size particles primarily affects their acceleration, whereas 
their Lagrangian velocity statistics are almost similar to those of tracers. With the exception of the studies by Klein et al. (2012) and Machicoane and Volk (2016), much less attention has been given to particles with $d_{p} \sim$ $\mathcal{O}(100) \eta \sim \mathcal{O}\left(10^{-1}\right) L_{i}$. The present study falls within this context.

Our experimental study was designed to examine the dispersion of largesized particles (compared to fluid tracers) in the entire volume of a rectangular open tank filled with water, in which turbulence was generated by moving a cylinder of diameter $2 R$ and length $L$ very similar to the depth of the tank along a periodic Lissajoux figure. In this setting, the turbulence is neither homogeneous nor isotropic, as boundary-layers at the walls and the free-surface are part of the volume of study, the cylinder is constantly interfering with the turbulent vortices, and the stirring is two-dimensional (2D), as no forcing is imposed in the direction along the axis of the cylinder. Particles were slightly negatively buoyant spheroids and their concentration in the fluid carrier was low. Their size was $d_{p} \sim R$. Consequently, particles were only to respond to eddies of size $\geq d_{p}$ such as those produced by the cylinder and its wake, while being unaffected by any eddy of size $<d_{p}$.

Following the works of Qureshi et al. (2007) and Bourgoin et al. (2011), we assumed that the Lagrangian particle velocity statistics were essentially similar to those of tracers. In other words, we assumed that the velocitybased properties of the turbulence could be inferred from the velocities of the finite-size particles. The energy dissipation rate $\varepsilon$ was thus derived from the particle motion. We found both a priori and a posteriori that this assumption was sensible. A priori, values of $\left\langle U^{2}\right\rangle$ and of $\varepsilon$ derived from the inertial range of spectra probed by the particles were estimated not to differ by 
more than 20\% from the corresponding exact fluid properties (see section 2.4). A posteriori, the regions in which the different dispersion regimes are displayed are within the temporal and spatial limits derived using the energy dissipation rate (see section 6.2). We stress however that the statistics of the experiments relies on a few particles in a bounded domain and is obtained from sampling in time via particle tracking.

The same stirring system and moving bodies were previously studied in a two-dimensional context with Smooth Particle Hydrodynamics (SPH) numerical models (Valizadeh and Monaghan, 2015; Monaghan, 2017; Monaghan and Mériaux, 2018a,b). In the presence of bodies, not all but many of the properties of the fluid could be estimated from the dynamics of those bodies.

For instance, the velocity auto-correlation times for the bodies and the fluid were found to be similar.

The structure of this paper is as follows. The laboratory experiments are described in $\S 2$, whereas the methodology of analysis is presented in $\S 3$. Since the experimental setup has never been described before, section $\S 4$ details the particle dynamics, from which we establish the inhomogeneity and anisotropy of the turbulence at the particle scale; Turbulence statistical properties are further inferred in section $\S 5$. Analysis of particle dispersion follows in section $\S 6$, and conclusions are gathered in $\S 7$.

\section{Laboratory experiments}

\subsection{Experimental setup}

The laboratory experiments were conducted in an Acrylic tank, $D=0.3$ $\mathrm{m}$ long ( $x$ direction), $W=0.3 \mathrm{~m}$ wide ( $y$ direction), and $0.5 \mathrm{~m}$ high that 
was filled with tap water up to a height $H=0.3 \mathrm{~m}$ ( $z$ direction). The tank itself was inserted into a metal wire frame secured to the experimental bench, at the top of which were fixed two electric actuator ball screw drives (SMC Pneumatics) mounted one over the other at right angles (Figure 1). These actuators were driven by two motors (Model AM8023 from BECKHOFF Automation), which were controlled by the TwinCAT software (BECKHOFF Automation). The two actuators were responsible for moving a cylinder in the tank in both the horizontal $(y)$ and vertical $(z)$ directions. The cylinder, which was hollow but capped at both ends, was hanging by a rigid rod of adjustable length from one of the actuators. Its centre was initially positioned at the tank mid-width, at a height of $0.15 \mathrm{~m}$. The cylinder had a radius $R=0.02 \mathrm{~m}$, and a length $L=0.298 \mathrm{~m}$, so it was only $2 \mathrm{~mm}$ shorter than the length of the tank $D$. It had been coated with a black film for visualisation purposes.

\subsection{Turbulence forcing}

Turbulence was generated in the water by forcing the cylinder to follow a cyclic Lissajous loop defined by

$$
\begin{aligned}
& y_{c}=y_{c}(0)+A \sin (2 \pi t / T), \\
& z_{c}=z_{c}(0) \pm A \sin (4 \pi t / T),
\end{aligned}
$$

where $y_{c}(0)$ and $z_{c}(0)$ are the initial $y_{c}$ and $z_{c}$ positions of the cylinder (see Figure 1). The amplitude $A$ was fixed at $0.075 \mathrm{~m}$, and the forcing period $T$ varied within the range $T=1.75-3 \mathrm{~s}$. The motion started initially either going down to the right as shown in Figure 1 or going up to the left (reverse). We 

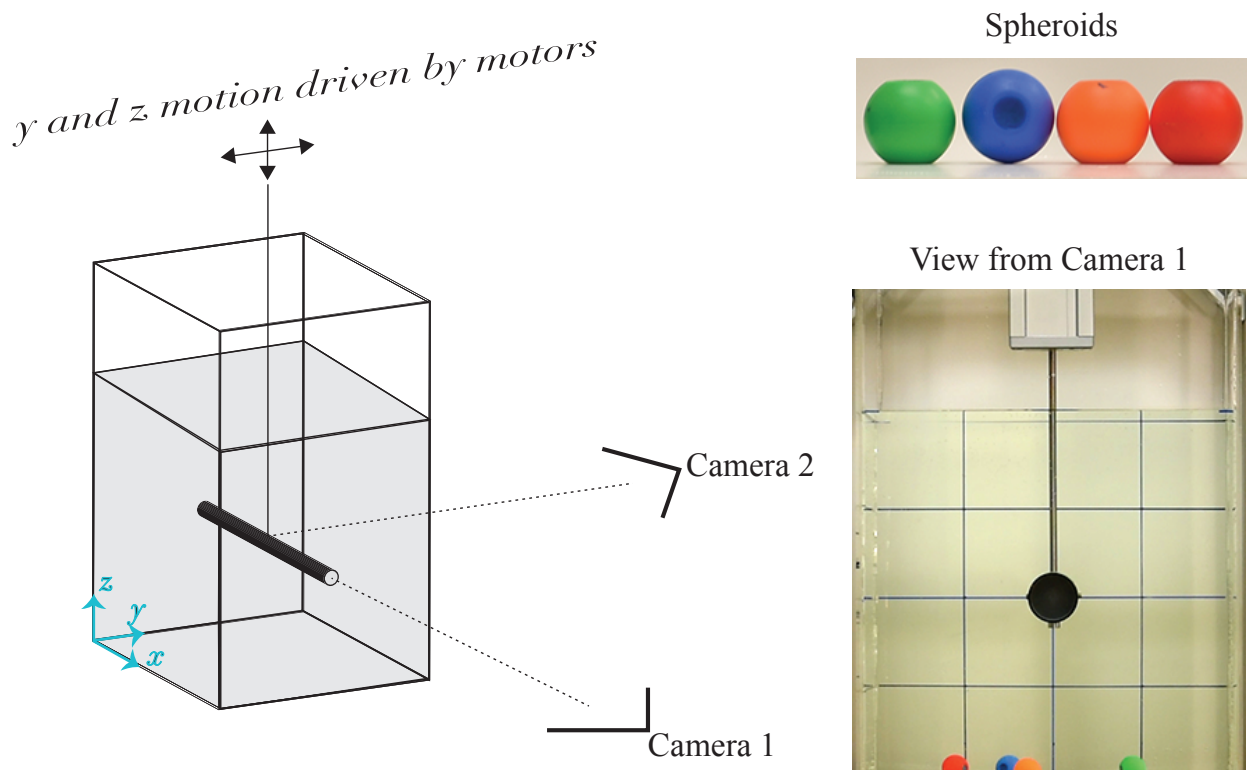

View from Camera 1

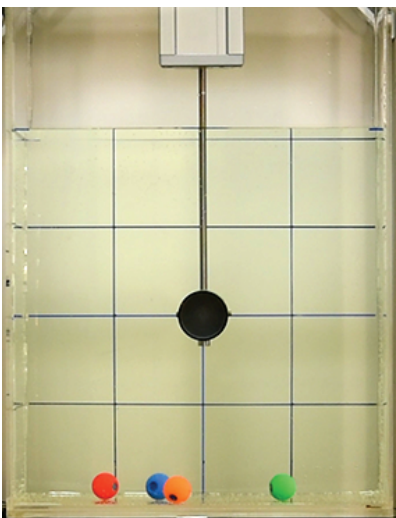

Cylinder motion

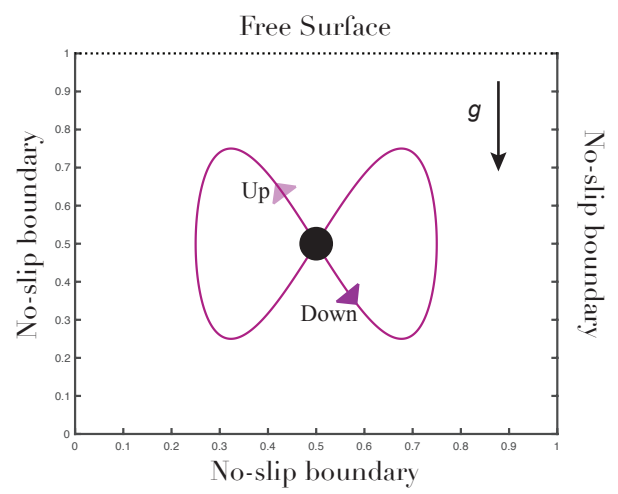

View from Camera 2

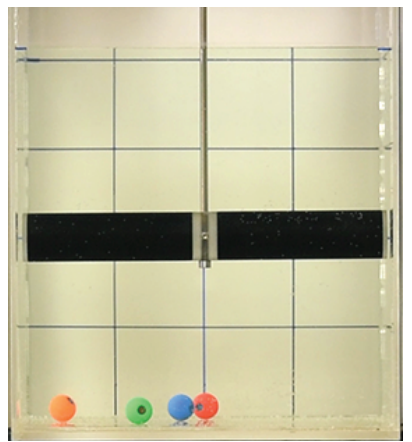

Figure 1: Experimental setup and coordinate system used, the Lissajous figure executed by the cylinder, and the finite-size particles used in the experiments. Note that the $x$ direction is along the cylinder, and the $y$ direction is horizontally across the cylinder. The $z$ direction is vertical. The cylinder motion executing a Lissajous figure starts either going down to the right or up to the left. The finite-size particles used in the experiments 1-10 are slightly oblate spheroids. 
identify those two initial directions of motion by down and up in Table 1. The velocity magnitude of the cylinder is given by $u_{c}=\sqrt{\left(d y_{c} / d t\right)^{2}+\left(d z_{c} / d t\right)^{2}}$. In the two directions of motion, the absolute maxima of the cylinder vertical velocity are located at mid-height in the tank at points given in dimensionless units by $\left(y_{c} / W, z_{c} / H\right)=(0.25,0.5),(0.5,0.5)$, and $(0.75,0.5) \mathrm{cm}$, and occur over a complete cycle at the times $2 \pi t / T=0, \pi / 2, \pi, 3 \pi / 2$, and $2 \pi$ (see Figure 1).

\subsection{Finite-size particles}

The finite-size particles, shown in Figure 1, were built from hollow plastic beads of different colours and are slightly oblate spheroids with an equatorial diameter $d_{p}=2.21 \mathrm{~cm}$ only $12 \%$ longer than the distance from pole to pole along the symmetry axis. Particles were filled with a single fishing weight and plasticine in order to be quasi-neutrally buoyant. The average density of the particles was $1015 \pm 10 \mathrm{~kg} \mathrm{~m}^{-3}$ giving an excess of density of the particles relative to the ambient water of $1.7 \%$.

\subsection{Experimental runs}

We report on eight experiments, which differed by the stirring period and direction of motion as detailed in Table 1. Across all experiments, the temperature of the water was $T_{w}=(20.9 \pm 1.1){ }^{\circ} \mathrm{C}$. Changes of the experimental conditions due the temperature change could be neglected as they were equivalent to a change of less than $0.1 \%$ in water density $\rho_{a}$, less than $7 \%$ in water dynamic viscosity $\mu_{a}$ and less than $1 \%$ in surface tension (see Vargaftik et al., 1983). 


\begin{tabular}{|c|c|c|c|c|c|}
\hline Experiment Id. & $\begin{array}{c}T_{w} \\
{ }^{o} \mathrm{C}\end{array}$ & $\begin{array}{c}T \\
\mathrm{~s}\end{array}$ & Initial motion & $N_{c}$ & $t_{s}$ \\
$\mathrm{~s}$ & \\
\hline 4 & 20.9 & 2.5 & Down & 128 & 51 \\
5 & 22.4 & 3 & Down & 118 & 72 \\
6 & 21.6 & 3 & Down & 102 & 78 \\
7 & 21.6 & 2 & Down & 100 & 86 \\
8 & 21.6 & 1.75 & Down & 121 & 86 \\
9 & 19.45 & 3 & Down & 110 & 74 \\
10 & 19.84 & 3 & Up & 85 & 56 \\
11 & 19.84 & 1.75 & Up & 99 & 96 \\
\hline
\end{tabular}

Table 1: Conditions for each run. $N_{c}$ refers to the total number of collisions between the particles and the cylinder over 100 cycles of its motion, which was manually counted by systematically inspecting all the video recordings. $t_{s}$ is the time at which the transient motion ends as defined in Appendix A. 
As we changed the stirring rate, the first normal mode of sloshing, at which a single peak and trough of a free surface wave oscillated between the $y$ vertical walls of the tank, was observed with a period of $T=1.75 \mathrm{~s}$, but only when the motion of the stirrer was initially going down to the right. When the motion of the stirrer was reversed, initially going up to the left, we did not detect any sloshing mode, which points to a different interaction with the free surface in the two directions of stirring. The observed sloshing period was also larger than the predicted sloshing period of a fluid in a rectangular tank (Ibrahim, 2005), estimated as

$$
T_{n}^{s}=\frac{2 \pi}{\sqrt{\frac{n g \pi}{W} \tanh \left(\frac{n \pi H}{W}\right)}},
$$

where $n$ is the mode number. When $n=1, T_{1}^{s}=0.6211 \mathrm{~s}$. This difference is likely due to the presence of the cylinder in the fluid, which acts as an obstacle.

Eight experiments were performed using the four particles previously described. We did not use any tracers to follow the fluid. The experiments were characterized by a set of dimensionless numbers and characteristic length and time scales, which are given in Table 2, with the underlying assumption that the impact of the particles on the velocity fluctuations in the fluid was small. In this regard, at least two dimensionless numbers have been found to be important for assessing the effect of particles on turbulence intensity: the volume fraction of particles in the fluid $\phi_{v}$ and the ratio of the particle size to the integral length scale of the turbulence $d_{p} / L_{i}$ (see for instance Balachandar and Eaton (2010) and Gore and Crowe (1989)). In our experiments, the 
volume fraction of particles in the fluid was

$$
\phi_{v}=\frac{v_{p}}{V_{f}}=4 \times \frac{\pi}{6}\left[\frac{d_{p}^{3}}{\left(D W H-\pi R^{2} L\right)}\right],
$$

where $V_{f}$, which coincides with the volume of measurement, is the total volume $D \times W \times H$ minus the volume of the cylinder $\pi R^{2} L$ and $v_{p}$ is the volume of the four particles

$$
v_{p}=4 \times\left(\frac{\pi}{6} d_{p}^{3}\right)
$$

$\phi_{v}$ was $8.4 \times 10^{-4}$ in the experiments, which is small. Such a volume fraction is, for example, below the threshold value of $1.4 \times 10^{-3}$, at which neutrallybuoyant Taylor-size spherical particles were shown to reduce by $15 \%$ the turbulent kinetic energy of the fluid (Bellani et al., 2012). Apart from $\phi_{v}$, in our experiments, $d_{p} / L_{i}=0.18-0.21$, which falls in the range where Gore and Crowe (1989) found that particles cause an increase in turbulence by not more than $20 \%$. So, regardless of whether there is a slight increase or a decrease in turbulence due to the particles, the values of $\phi_{v}$ and $d_{p} / L_{i}$ in our study imply that the modulation of the turbulence due to the particles should be limited.

Additionally, our study shares dynamic similarities with the studies by Bellani et al. (2012) $\left(\phi_{v} \ll 1 ; d_{p} / L_{i}=0.11\right)$, Qureshi et al. $(2007)\left(\phi_{v} \ll 1\right.$; $\left.d_{p} / L_{i}=0.02-0.10\right)$ and Bourgoin et al. (2011) $\left(\phi_{v} \ll 1 ; d_{p} / L_{i}=0.04-0.12\right)$, which all showed little impact of the particles on the velocity fluctuations in the fluid. It however departs from these studies by its ratio of the particle size to the Kolmogorov scale $d_{p} / \eta$, which is of order $\mathcal{O}(100)$ and therefore greater than the ratios used in the previous studies, $d_{p} / \eta=[7,30]$. In our study, the departure of the acceleration variance of particles from that of tracers in the 
inertial range $\mathcal{R}=\left\langle a^{2}\right\rangle_{\text {particle }} /\left\langle a^{2}\right\rangle_{\text {fluid }}$ is estimated to be in the interval [0.160.22], following equations 2 and 3 of Qureshi et al. (2007), which predict $\mathcal{R} \sim$ $6\left(d_{p} / \eta\right)^{-2 / 3}$. The impact of particle inertia on the acceleration is therefore substantial (80\%). This impact on the acceleration does not however imply a substantial impact of the particles on the velocity fluctuations in the fluid, as shown previously by Qureshi et al. (2007) and Bourgoin et al. (2011) when $d_{p} / \eta=[7,30]$. The time integration by which the velocity is obtained from the acceleration acts like a low-pass filter $\left(\Phi(\omega)=\omega^{2} E(\omega)\right.$, where $\Phi(\omega)$ is the acceleration frequency spectrum), making the velocity be dominated by lower frequencies, less affected by inertia, compared to the acceleration. In practice, the relation between the velocity variance of the particles $\left\langle u^{2}\right\rangle$ and the energy spectrum $E(k)$ can be defined as for tracers, but integrated over a narrower range of wavelengths that excludes scales smaller than the particle size, that is $(3 / 2)\left\langle u^{2}\right\rangle=\int_{2 \pi / L_{i}}^{2 \pi / d_{p}} E(k) d k$. This truncation of the spectrum of the turbulence reflects the fact that particles do not respond to scales of fluid motion smaller than their own size. Calculations using the model spectrum adopted by Teixeira and Belcher (2000) for a range of Reynolds numbers of the order of magnitude of those used in the experiments, $R e=\left[10^{2}-10^{4}\right]$, actually indicate that this truncation does not lead to an underestimation of the velocity variance of the fluid motion by more than about $20 \%$. Similarly, the estimate of $\varepsilon$ captured by the particles from the slope of the inertial range is expected to be even more accurate, since there is a factor of $\sim 5$ between the integral length scale $L_{i}$ and the scale of the particles $d_{p}$, which allows a sufficient window of motions in the inertial range to be well resolved by the particles. However, the inertial range detected in this way is necessarily 
relatively narrow, as will be confirmed later. These arguments allow us to extend the domain of validity of the conclusions drawn by Bourgoin et al. (2011) and Qureshi et al. (2007) for $d_{p} / \eta \sim 30$ to $d_{p} / \eta \sim \mathcal{O}(100)$.

The experiments were characterized by a set of dimensionless numbers and characteristic length and time scales, which are given in Table 2. These dimensionless numbers and scales depend on the energy dissipation rate $\varepsilon$, whose estimate will be thoroughly detailed in section 5 .

\begin{tabular}{c|ccccccccc} 
Experiment Id. & $U^{\prime}$ & $\begin{array}{c}\varepsilon \\
\mathrm{cm} \mathrm{s}^{2} \mathrm{~s}^{-3}\end{array}$ & $\begin{array}{c}L_{i} \\
\mathrm{~cm}\end{array}$ & $\begin{array}{c}\tau_{e} \\
\mathrm{~s}\end{array}$ & $\begin{array}{c}R e \\
\mathrm{~cm}_{\lambda}\end{array}$ & $\begin{array}{c}\lambda \\
\mathrm{mm}\end{array}$ & $\mu \mathrm{m}$ & $\mathrm{ms}$ \\
\hline 4 & 7.0 & $31.0 \pm 2.5$ & 11.0 & 1.6 & 7682 & 339 & 4.9 & 133.8 & 18.0 \\
5 & 5.6 & $17.1 \pm 1.6$ & 10.2 & 1.8 & 5693 & 292 & 5.2 & 155.3 & 24.2 \\
6 & 5.7 & $15.4 \pm 1.4$ & 12.1 & 2.1 & 6942 & 323 & 5.5 & 159.8 & 25.5 \\
7 & 8.6 & $55.9 \pm 4.3$ & 11.3 & 1.3 & 9733 & 382 & 4.4 & 115.7 & 13.4 \\
8 & 10.3 & $108.3 \pm 7.6$ & 10.2 & 1.0 & 10586 & 398 & 3.9 & 96.9 & 9.6 \\
9 & 5.8 & $16.5 \pm 1.5$ & 11.5 & 2.0 & 6636 & 316 & 5.5. & 157.0 & 24.6 \\
10 & 5.2 & $11.8 \pm 1.3$ & 11.9 & 2.3 & 6206 & 306 & 5.9 & 170.9 & 29.1 \\
11 & 9.6 & $73.4 \pm 5.9$ & 12.1 & 1.3 & 11649 & 418 & 4.3 & 108.1 & 11.7 \\
\hline
\end{tabular}

Table 2: Experimental scales and dimensionless numbers. $U^{\prime}=$ $\sqrt{\left(\left\langle U_{x}\right\rangle^{2}+\left\langle U_{y}\right\rangle^{2}+\left\langle U_{z}\right\rangle^{2}\right) / 3}$ is the particle velocity averaged over components. The energy dissipation $\varepsilon$ represents an average of the estimates from the Lagrangian velocity structure function, the energy spectrum and the longitudinal structure function. The integral length scale is given by $L_{i}=U^{\prime 3} / \varepsilon$ and the eddy turn-over time by $\tau_{e}=L_{i} / U^{\prime}$. The Reynolds number is estimated as $R e=L_{i} U^{\prime} / \nu$ and the Reynolds number based on the Taylor micro-scale $\lambda$ is estimated as $R_{\lambda}=\lambda U^{\prime} / \nu$ with $\lambda=\sqrt{15 U^{\prime 2} \nu / \varepsilon}$. The Kolmogorov length and time scales are respectively $\eta=\left(\nu^{3} / \varepsilon\right)^{1 / 4}$ and $\tau_{\eta}=(\nu / \varepsilon)^{1 / 2}$. 


\section{Analysis methodology}

\subsection{Particle tracking}

The experiments were recorded during $300 \mathrm{~s}$ from two sides by cameras in video mode providing one plane view across the cylinder axis, and another along the cylinder axis. As we varied the period of the cylinder motion from $3 \mathrm{~s}$ to $1.75 \mathrm{~s}$, the recording covered a minimum of 100 cycles (18,000 frames) to a maximum of 171 cycles, implying that the number of samples slightly differs across the 11 experiments when calculating statistical quantities. The videos were produced at a resolution of $1920 \times 1080$ pixels and at a number of frames per second $n_{f}=59.94$ frames $/ \mathrm{s}$. Note that at this sampling rate we did not expect to resolve the dissipative turbulence range as $T / n_{f} \sim \mathcal{O}(10) \tau_{\eta}$, where $\tau_{\eta}$ is the Kolmogorov time scale (see Table 2). The two recordings were first synchronized using the frame at which the cylinder started to move. A camera calibration was performed using the landmarks of grids that had been drawn on the sides of the tank to measure the 3D coordinates of the particles. Particles and cylinder were tracked based on their coloured pixels, and we followed the centre of the finite-size spheres as shown in Figure 2a. The set of centre particle positions over time $(X(t), Y(t), Z(t))$ defined the particle trajectory as shown Figure 2b. We did not track the particle orientations. To compute the velocity of the spheres, a monotonic cubic spline was fitted to the $(X, Y, Z)$ particle positions for the purpose of applying a first-order differentiation. 
a)

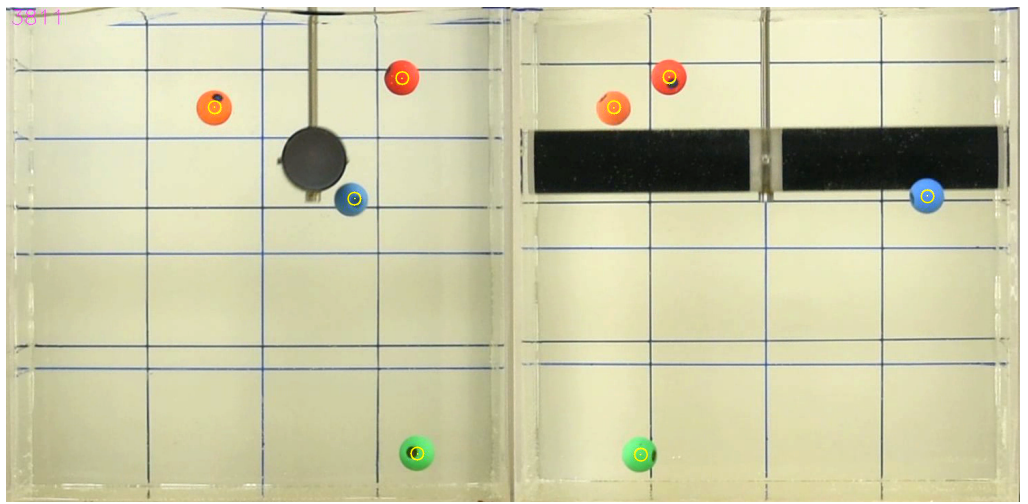

b)
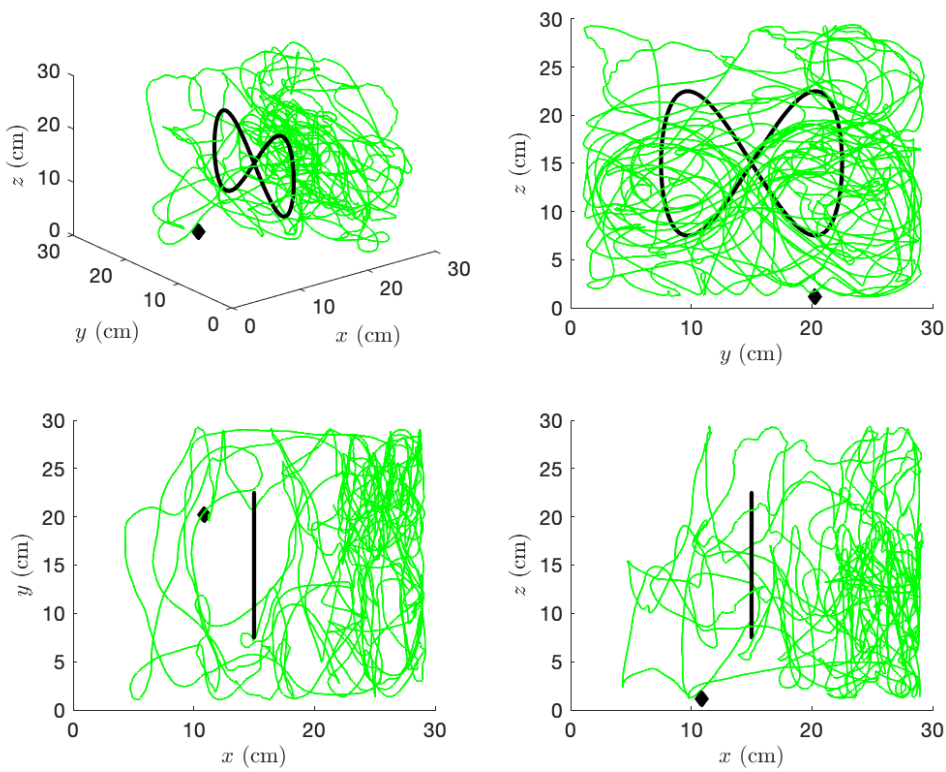

Figure 2: a) Three-dimensional tracking of the particles from the two side views taken by the cameras. The background lines are the grids used for 3D geo-referencing and camera calibration. The yellow circles mark the centre of the finite-size particles identified by particle tracking. b) Reconstructed trajectory. The example shows that of the green particle in experiment 5 seen in different views, including looking parallel and perpendicular to the axis of the cylinder and from the top. The black line represents the path of the cylinder in each plane $(y, z),(x, y)$ and $(x, z)$. For visibility reasons, the cylinder position along the $x$ direction is simply shown at the centre $x=15 \mathrm{~cm}$. The black diamond indicates the initial position of the particle. 


\subsection{Statistical analysis}

Statistical analysis was used to interpret the data. We first checked the equivalence of the 4 particles, and assessed the role of the collisions and transient behaviour of the particles. Appendix A and Appendix B give full details on the existence of a transient period and its duration, and on the lack of impact of the collisions on the particle velocity statistics. As a result, in data sets containing positions and velocities, the data corresponding to the transient were removed; velocities of the resulting data sets were not filtered for the collisions; and data sets of the four particles were assimilated into a single set for analysis, such as for estimating Probability Density Functions (PDF). Besides, spatial statistical analyses were performed after subdividing the entire (undisturbed) volume of fluid $V=H \times D \times W=(30 \mathrm{~cm})^{3}$ into $15^{3}$ cells of dimensions $V_{c}=(2 \mathrm{~cm})^{3}$, i.e. the cubic volume occupied by a particle. In a cell $(i, j, k)$ of central position $\left(x_{i}, y_{j}, z_{k}\right)$, we evaluated the count of particles $N(i, j, k)$, and the velocity $\boldsymbol{U}(i, j, k)$. We note that all the cells close to a boundary will be statistically different from interior cells because the finite-size of the particles implies that their centres are at a distance of at least $1 \mathrm{~cm}$ from the lateral walls or bottom of the tank. In other words, compared to an interior cell, only half a cell effectively contributes to the statistics when it is bounded by a tank wall, because in those cells the centre of a particle is constrained to take a position in only half of its volume (farther from the boundary). 


\section{Dynamics of the particles}

\subsection{Ensemble particle localization}

An insight into the ensemble wandering of particles in the tank was first gained by analyzing the percentage of fluid volume that was never visited by the four particles over the duration of each experiment excluding the transient. Wandering of particles increases as the stirring rate increases, as shown in Figure 3. An exponential fit to the data further indicates that this study uses a range of stirring rates that achieves reasonable excursion of the particles. Increasing further the stirring rate would have increased the particle wandering but it was technically not possible due to the torque limit of the actuators.

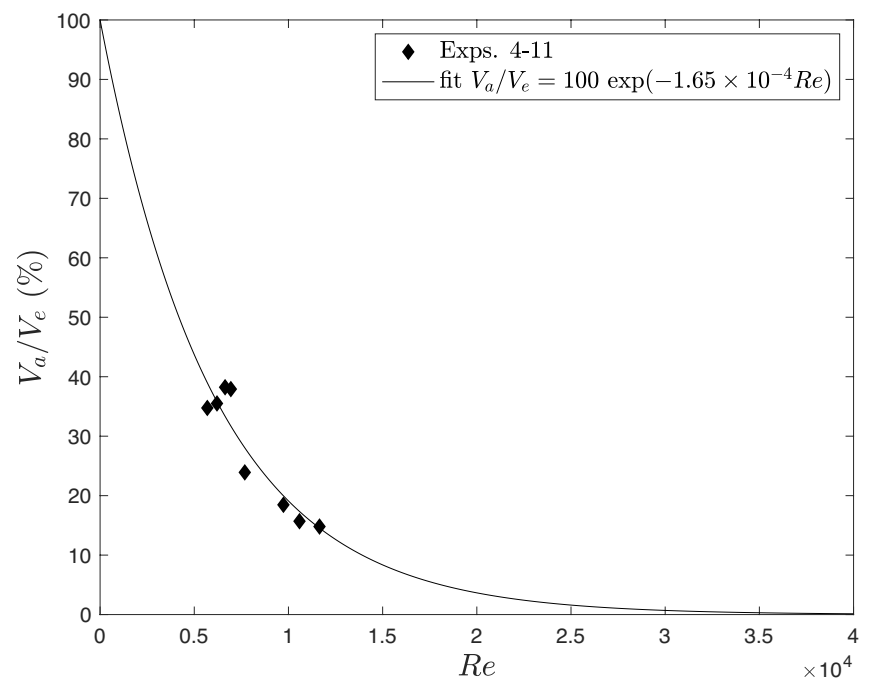

Figure 3: Dimensionless volume $V_{a} / V_{e}$ (in \%) never visited by any of the four particles as a function of Reynolds number $R e$ for experiments $4-11$. Note that $V_{a}$ has been normalized here using the volume accessible to the particles $V_{e} \sim V\left(1-d_{p} / W\right)^{2}$. 
The increase in wandering with the stirring rate especially applies to the particle excursion in the $z$ (vertical) direction. As shown in Figure 4, at low stirring rate, the PDFs of the $Z$ particle coordinate are higher in the lower half of the tank regardless of the direction of stirring. At high stirring rate, however, there is much less vertical disparity between the two directions of cylinder motion. The increase in stirring velocity helps to counteract the slight negative buoyancy of the particles, whose presence in the upper half of the tank is facilitated by the more vigorous vortices.
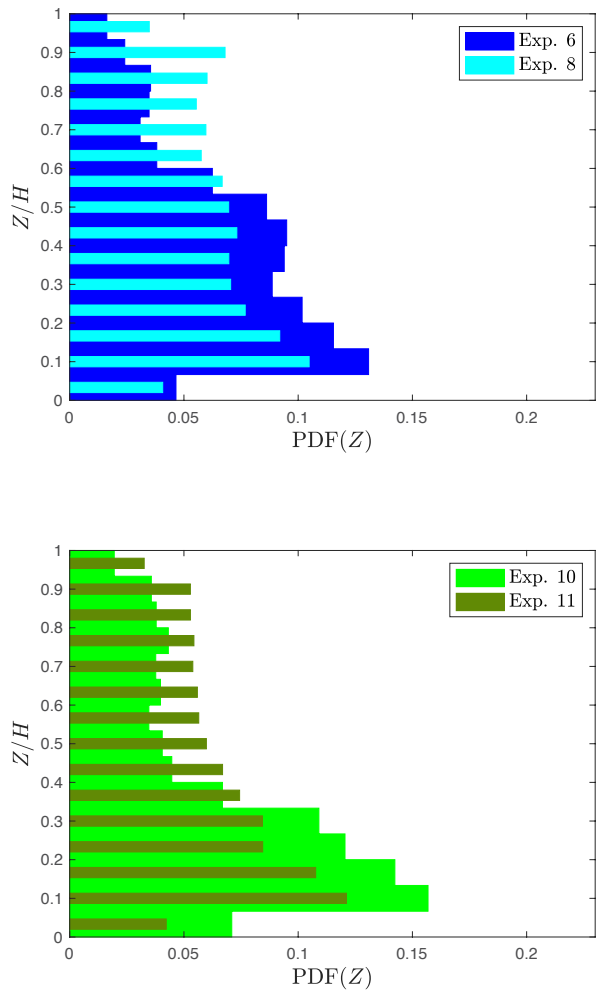

Figure 4: Probability Density Functions (PDFs) of particle dimensionless vertical coordinate $Z / H$ in experiments $6 \& 8$, and $10 \& 11$. The stirring is weaker in experiments $6 \&$ 10 than in experiments $8 \& 11$. 


\subsection{Inhomogeneous and anisotropic flow inferred from the particles}

While the stirring is obviously anisotropic, the flow inferred the particle motion is also influenced by the anisotropy of the forcing. This is shown in Figure 5 by the distributions of the direction cosines of the particle velocity vectors calculated as $\operatorname{cosine}\left(\alpha_{i}^{j}\right)=U_{i}^{j} /\left|\boldsymbol{U}^{j}\right|$, where $i$ refers to components $x, y$ and $z$ and $j$ runs from 1 to four times the number of frames recorded between the end of the transient and the end of the experiment. Regardless of the direction and intensity of stirring, the direction cosines are more uniformly distributed in the $y$ and $z$ directions, whereas in the $x$ direction the distribution is non-uniform and peaks around zero, indicating a preferred direction of the velocity vectors normal to the $x$ axis. However, the histograms in Figure 5 also show that the motion is far from being perfectly two-dimensional (which would correspond to a Dirac function $\delta(0)$ for cosine $\left(\alpha_{x}\right)$ ).

a)

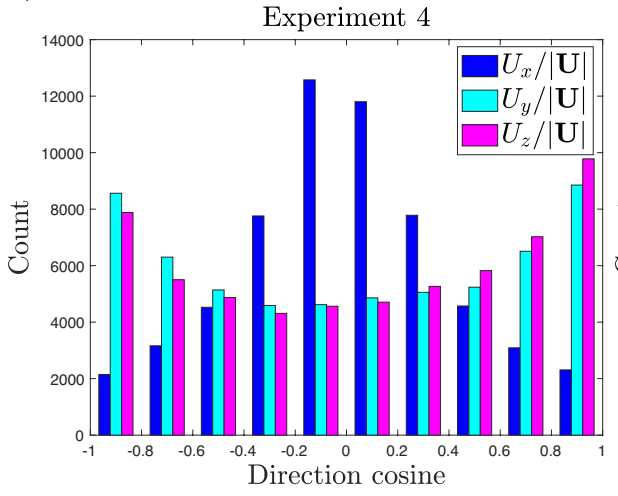

b)

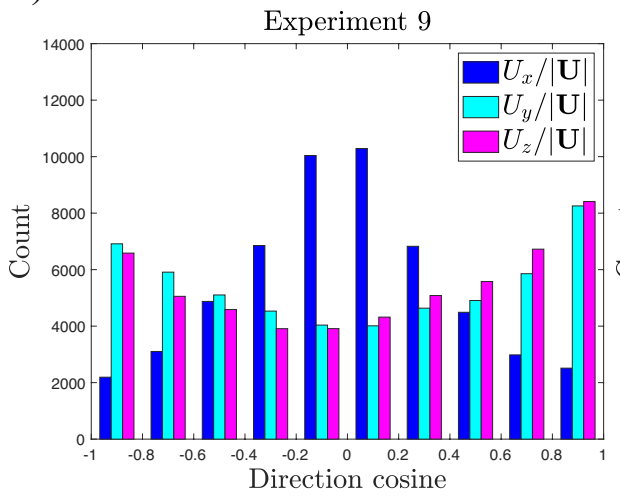

c)

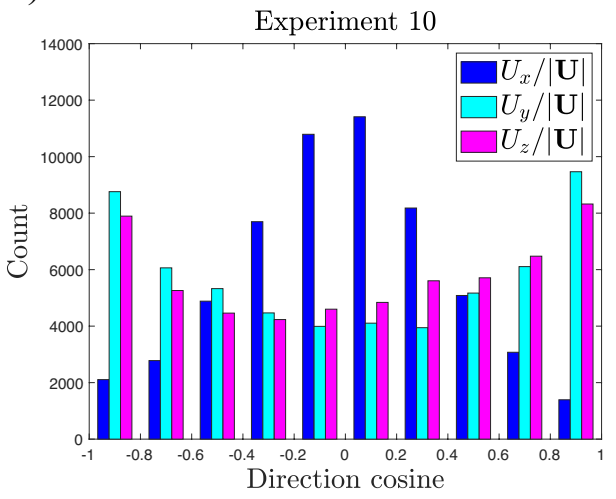

Figure 5: Raw histograms of the direction cosines of the particle velocity for experiments a) 4, b) 9 and c) 10. Experiments 4, 9 and 10 exemplify experiments at different directions and/or intensity of stirring. 
$x=15 \mathrm{~cm}$
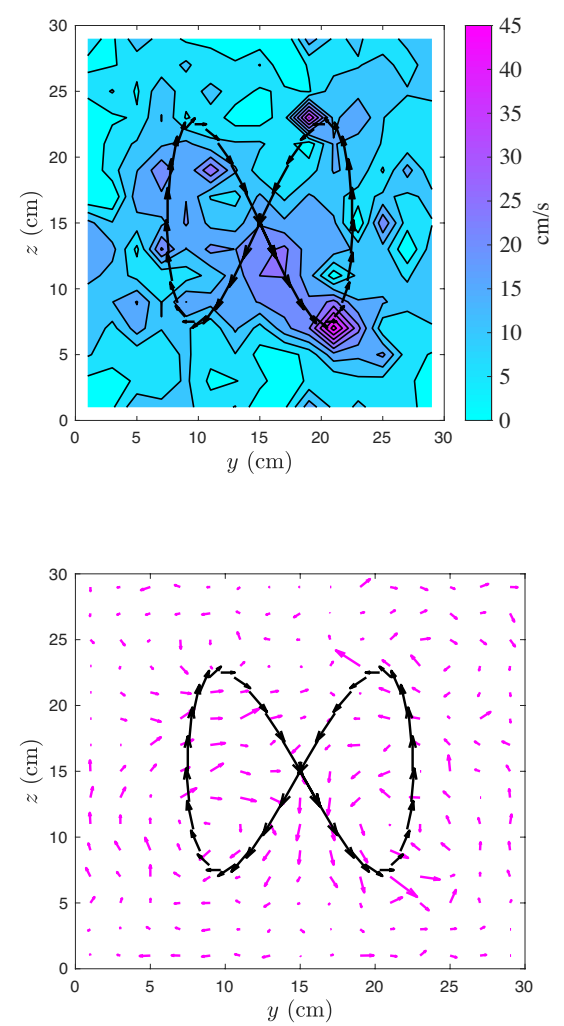
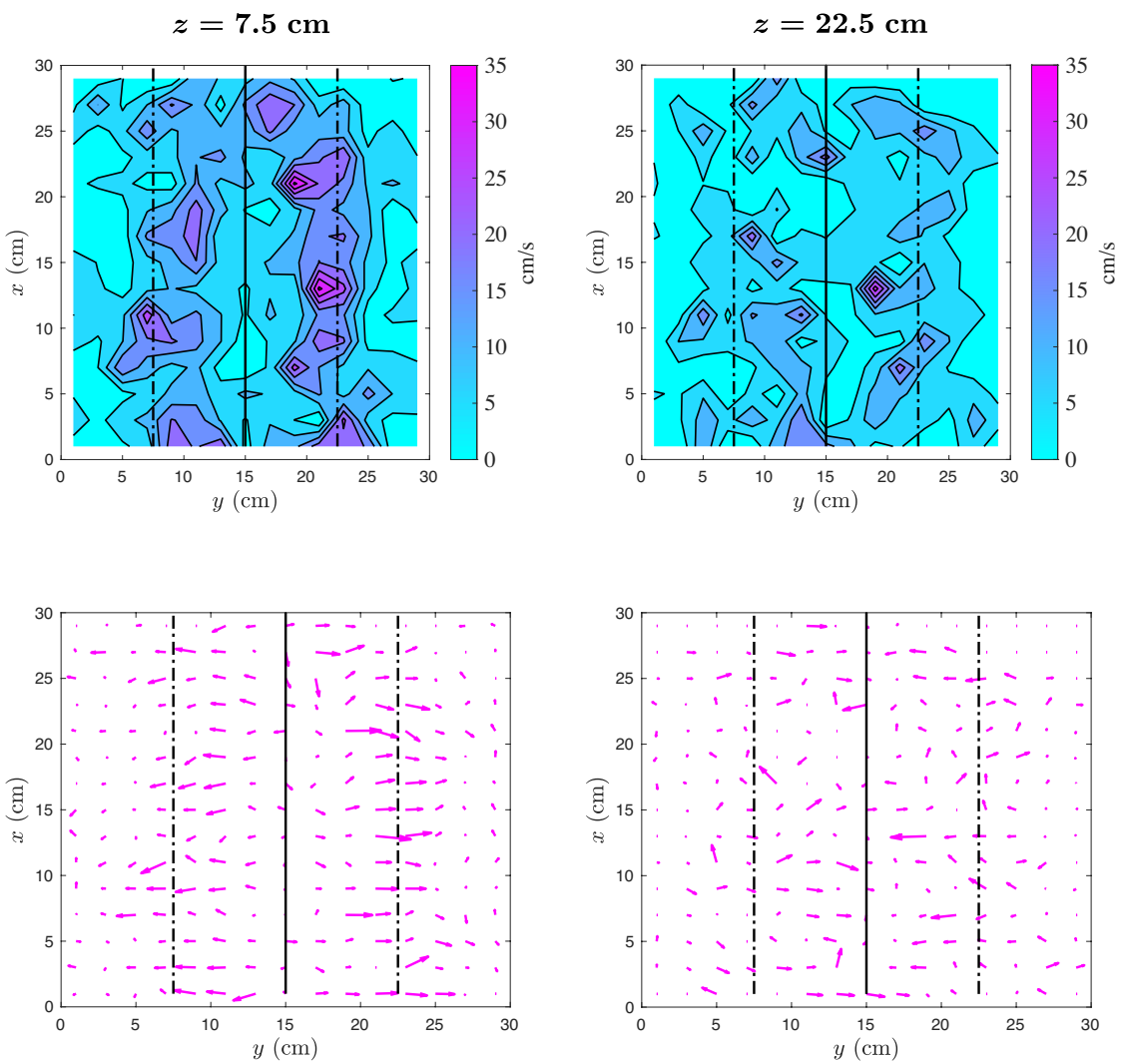

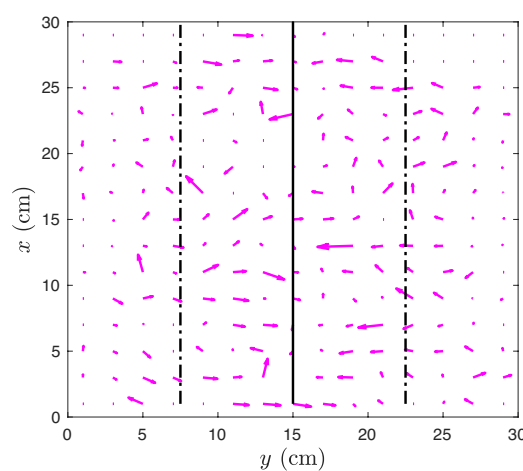

Figure 6: Contour maps of the flow speed (top frames) and corresponding velocity vector field (lower frames) in experiment 8. The black lines delimit the path or region reached by the cylinder. The colour scale indicates the magnitude of the flow speed. The size of the velocity vectors in the flow fields has been scaled for visualisation purposes.

Aside from preferential directions normal to the $x$ axis, consistent the anisotropy discussed in Figure 5, the velocity fields in the interior of the 

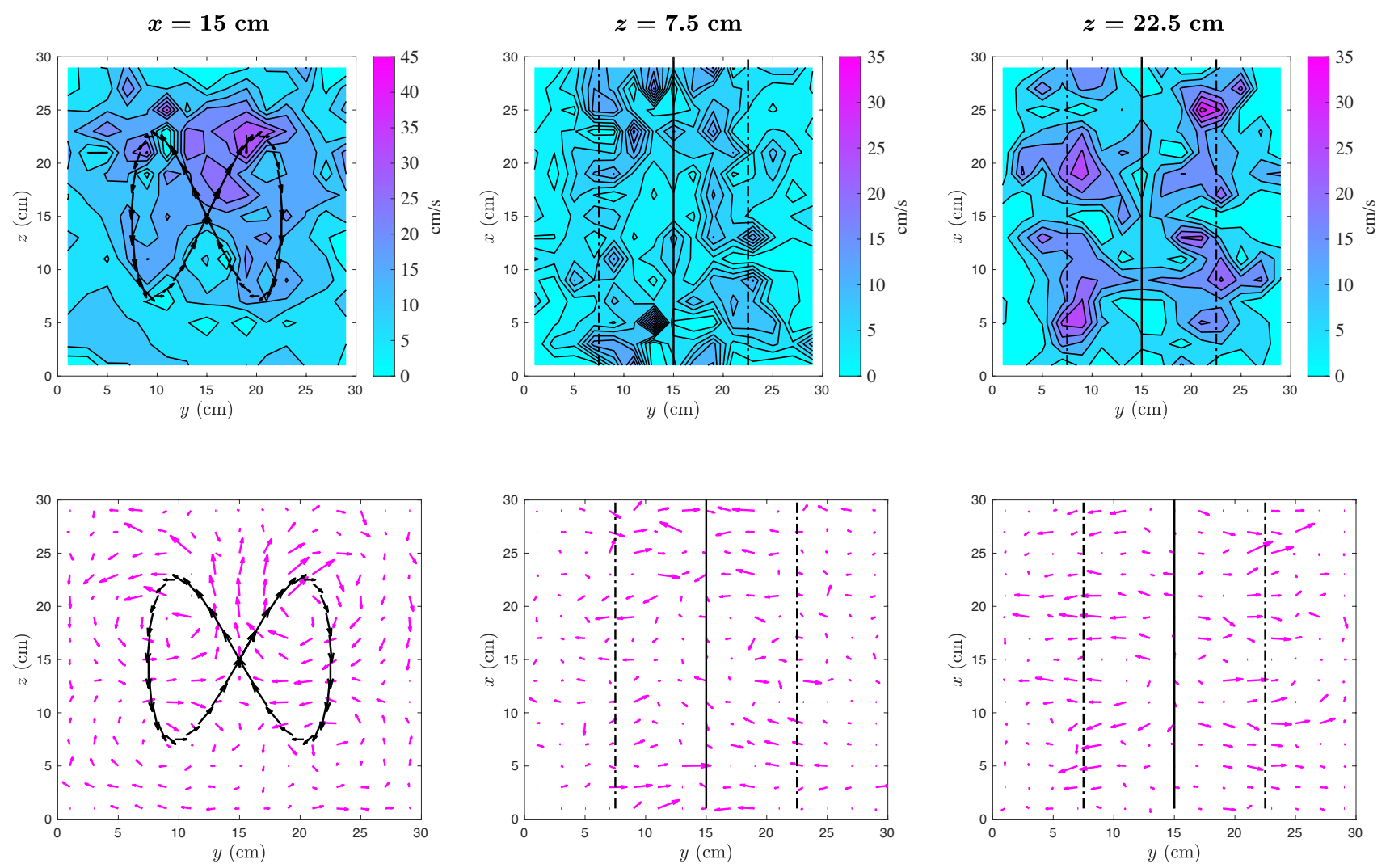

Figure 7: Contour maps of the flow speed (top frames) and corresponding velocity vector field (lower frames) in experiment 11. The black lines delimit the path or region reached by the cylinder. The colour scale indicates the magnitude of the flow speed. The size of the velocity vectors in the flow fields has been scaled for visualisation purposes.

measurement volume are quite inhomogeneous. They comprise areas of high velocity being essentially located along the cylinder path, which contrasts with areas of low velocity, especially close to boundaries (i.e. walls, and the free surface). However, differences in inhomogeneity exist between the two directions of stirring, especially in the vertical direction. In the case of stirring with initial downward cylinder motion, large-scale vortices are most 
active in the lower half of the tank, whereas for stirring with initial upward cylinder motion, large-scale vortices are most active in the upper half of the tank, as shown by Figures 6 and 7. Interestingly, for stirring with initial upward cylinder motion, large-scale vortices also exist in the lower half of the tank, although they have a weaker intensity, but there are almost no vortices in the upper half of the tank in the case of stirring with initial downward cylinder motion.

\subsection{Individual trajectories}

While the global flow features seem to point to a reasonably efficient wandering of the particles in the tank, although constrained by the looping motion of the cylinder in the $(y, z)$ plane perpendicular to the cylinder axis, more complex behaviour emerges when looking at the particles individually. In particular, substantial variability between different particles is found regarding their excursions in the $x$ (i.e. along-axis) direction, in which the cylinder does not generate any direct forcing motion. This effect seems to prevent individual particles from crossing the whole domain along $x$, leading to particles being strikingly confined, as shown in Figure 2b. The observed particle confinement in the $x$ direction is consistent with the anisotropic flow dominated by motion in the $(y, z)$ plane, as shown in Figure 5 , and is a manifestation of the conservation of the angular momentum perpendicular to that plane. An uneven localization along the $x$ direction is observed regardless of the stirring rate and direction, as shown by the $(y, z)$ plane-averaged residence times as a function of $x$ in Figure 8. We will see that this contributes to a differentiation of the particle pairs during dispersion. 

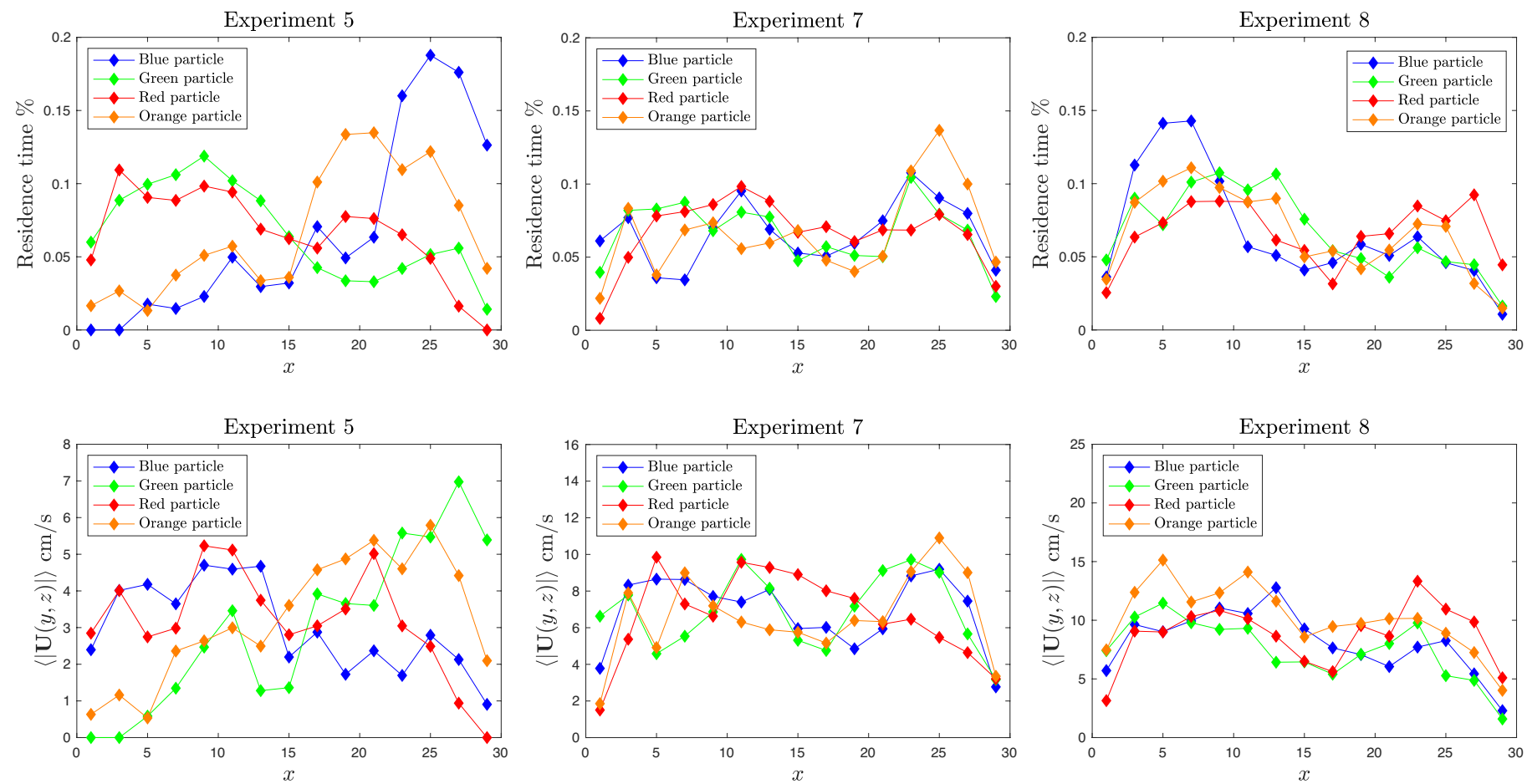

Figure 8: Top frames: $y-z$ plane-averaged residence time of the particles along the $x$ direction, respectively, for experiments 5, 7 and 8. Bottom frames: corresponding $y-z$ plane-averaged velocity magnitude along the $x$ direction in the same experiments. Note that the particle residence time anti-correlates well with the particle plane-averaged velocity: where the averaged velocities appear larger, the residence time is lower, and vice-versa. This is to be expected, since the residence time should scale inversely to the flow velocity. 


\section{Statistical properties}

As was mentioned in the Introduction, the time $t_{0}$ delimiting the ballistic and super-diffusive regimes in 3D dispersion is defined in terms of the energy dissipation rate, $\varepsilon$. This requires obtaining an estimate of this quantity. To do so, we used both the Lagrangian and Eulerian frameworks. The Lagrangian velocity structure function $D$ and frequency spectrum $E$ are strictly tensors of order 2 because the anisotropy of the large-scale flow is also present in the smaller-scale fluctuations of the particles. These tensors are respectively defined as:

$$
D_{i j}(\tau)=\left\langle\delta U_{i}(\tau) \delta U_{j}(\tau)\right\rangle
$$

where $\delta U_{i}(\tau)=U_{i}(t+\tau)-U_{i}(t)$ (Monin and Yaglom, 2013; Mordant et al., 2003) and

$$
E_{i j}\left(\omega_{k}\right)=\frac{\delta t}{2 \pi}\left[2\left(\sum_{j=1}^{n-1} R_{i j}(j \delta t) \cos \left(\omega_{k} t_{j}\right)\right)+R_{i j}(0)+R_{i j}(n \delta t)\right],
$$

taken at equal sampling intervals of size $\delta t$ with $\omega_{k}=k \pi / n \delta t(k=0, \pm 1, \ldots \pm$ $n$ ) and $R_{i j}(\tau)=\left\langle U_{i}(t) U_{j}(t+\tau)\right\rangle$ (Yeung and Pope, 1988). Here we only evaluated the $D_{i i}$ and $E_{i i}$ components of these tensors together with an estimate of the trace of $D$ defined as $\operatorname{Tr}(D)=1 / 3 \sum_{i} D_{i i}$. Alternatively, in the Eulerian framework, we estimated the dissipation rate from the secondorder longitudinal structure function $C_{2}(l)$ assuming that the instantaneous velocity at a similar time $t$ of two particles respectively with positions $\boldsymbol{x}$ and $\boldsymbol{x}+\boldsymbol{l}$ coincides with the local Eulerian velocity field. $C_{2}(l)$ is thus defined by

$$
C_{2}(l)=\left\langle([\boldsymbol{U}(\boldsymbol{x}+\boldsymbol{l}, t)-\boldsymbol{U}(\boldsymbol{x}, t)] \cdot \boldsymbol{l} / l)^{2}\right\rangle,
$$


where $\boldsymbol{U}(\boldsymbol{x}, t)$ and $\boldsymbol{U}(\boldsymbol{x}+\boldsymbol{l}, t)$ are the velocities of a pair of particles at time $t$ and positions $\boldsymbol{x}$ and $\boldsymbol{x}+\boldsymbol{l}$. Such an evaluation of $C_{2}(l)$ is similar to that used in Valizadeh and Monaghan (2012) with SPH tracer-like particles, but for particle separations $l>d_{p}$, where the particle velocities can be representative of the flow (Qureshi et al., 2007; Bourgoin et al., 2011). $C_{2}(l)$ was further averaged over the six pairs of particles.

Figure 9 shows the compensated Lagrangian velocity structure function $D_{i i}(\tau) /\left(C_{0} \tau\right)$, frequency spectrum $\pi E_{i i}(\omega) \omega^{2} / C_{0}$ and the second-order longitudinal structure function $\left(C_{2}(l) l^{-2 / 3} / C_{K}\right)^{3 / 2}$. All three statistical properties were compensated with the dimensional expression given by the classical Kolmogorov theory in the inertial range (see section 1). We used the scaling constants $C_{0}=5$ and $C_{K}=2.01$, which have been associated with threedimensional turbulence, including in anisotropic contexts (Ouellette et al., 2006). All three statistical quantities consistently show a plateau even if the plateau is better developed for the energy spectrum and the longitudinal structure function than for the Lagrangian velocity structure function. Values of the compensated functions at their plateaus were used to estimate $\varepsilon$ for each experiment. In practice, the value of $\varepsilon$ inferred from the compensated Lagrangian velocity structure function $D_{i i}(\tau) /\left(C_{0} \tau\right)$ was simply taken as being an average of the maximum compensated values for the three velocity components at $\tau=0.2-0.3$ as the structure function did not have a well-defined plateau. For the frequency spectrum $\pi E_{i i}(\omega) \omega^{2} / C_{0}$ and the second-order longitudinal structure function $\left(C_{2}(l) l^{-2 / 3} / C_{K}\right)^{3 / 2}$, which had better-defined plateaus, values of $\varepsilon$ were calculated as an average of the means of the compensated values over the frequency range $w=[4,10] \mathrm{s}^{-1}$ 
a)
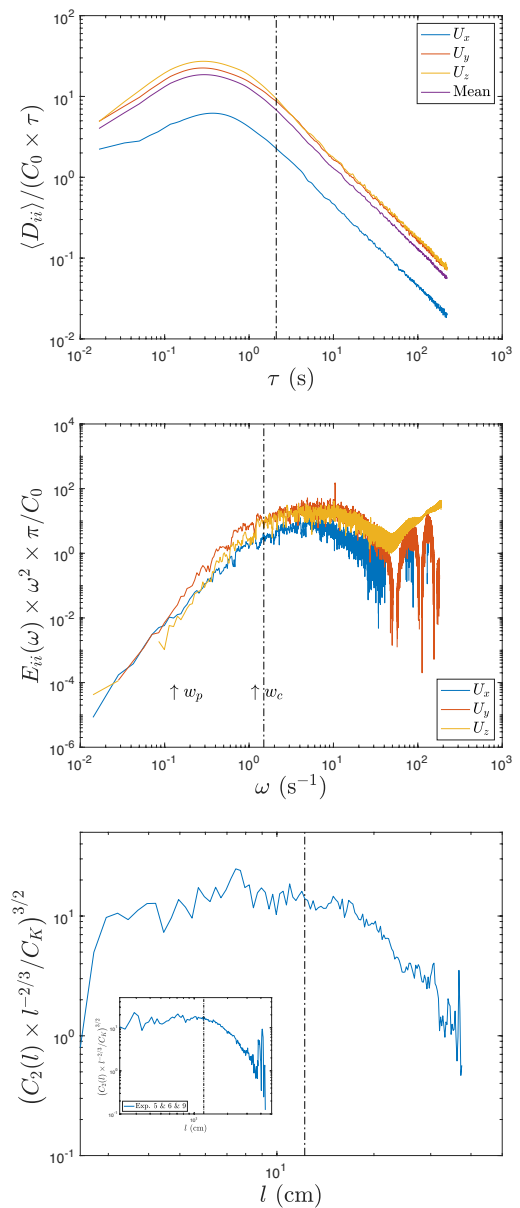

b)
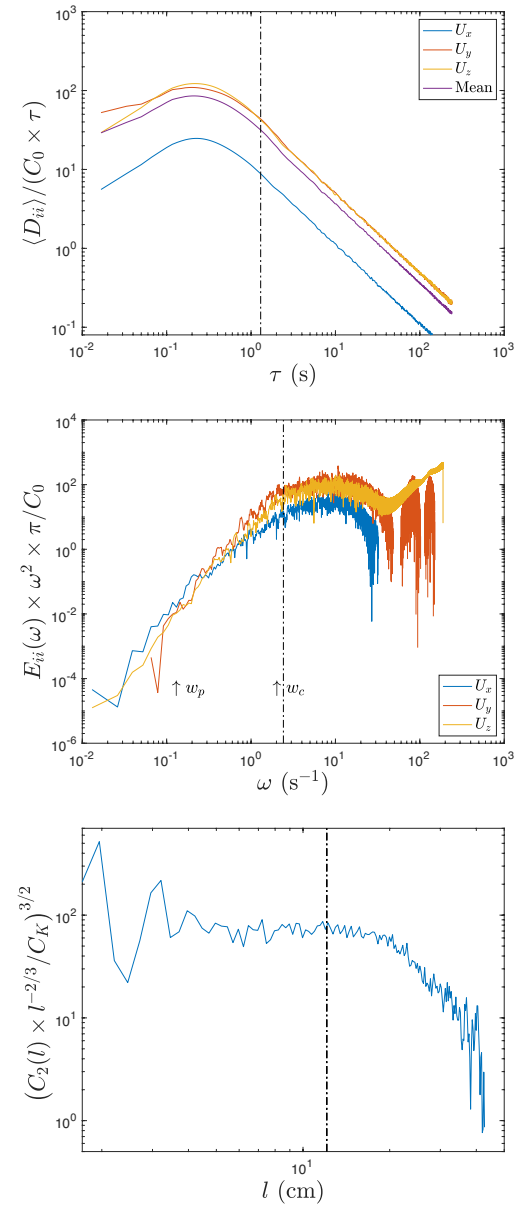

Figure 9: Compensated Lagrangian velocity structure function, frequency spectrum and second-order longitudinal structure function in a) experiment 6 and b) experiment 11 . The values $w_{c}=\pi / T$ and $w_{p}=\pi / t_{p}$, where $t_{p}$ is the particle relaxation time, are the frequency of the particles and cylinder, respectively. The inset in the figure for the longitudinal structure function $\left(C_{2}(l) l^{-2 / 3} / C_{K}\right)^{3 / 2}$ of experiment 6 is the same structure function but evaluated using the three experiments $5,6 \& 9$, giving a smoother compensated function as the statistical sampling is increased. The vertical dashed lines indicate the limits $\tau=\tau_{e}$, $\omega=\pi / \tau_{e}$ and $l=L_{i}$. In experiment $6, \varepsilon$ derived from the three functions gives a mean value with standard error, $\varepsilon=(15.39 \pm 1.40) \mathrm{cm}^{2} \mathrm{~s}^{-3}$. In experiment $11, \varepsilon$ derived from the three functions gives a mean with standard error, $\varepsilon=(73.38 \pm 5.85) \mathrm{cm}^{2} \mathrm{~s}^{-3}$. 
and length range $l=[5,10] \mathrm{cm}$, respectively, for the three velocity components. We note that these averages for the Lagrangian velocity structure function, $1 / 3 \sum_{i} D_{i i}(\tau)$, and the frequency spectrum, $1 / 3 \sum_{i} E_{i i}(\tau)$, represent the isotropic decomposition of $D$ and $E$. Finally, as the standard errors of the mean of values of $\varepsilon$ inferred from $D, E$ and $C_{2}$ ranged only between 4 and $6 \%$, we obtained a final estimate of $\varepsilon$ as the average of these three estimates. The resulting value of $\varepsilon$ was then used to calculate the associated flow scales and dimensionless numbers (see Table 2). The time $\tau$ corresponding to the maximum of the compensated Lagrangian velocity structure function $D(\tau)$ is reasonably consistent with the values of the eddy turn-over time $\tau_{e}$ given in Table 2. Similarly, the lower limit $\omega$ of the plateaus of the frequency spectrum $E(\omega)$ reasonably agrees with the frequency $\omega_{e}=\pi / \tau_{e}$. In the spatial domain, the region of the function $C_{2}(l)$ between the lags $l=4 \mathrm{~cm}$ and $l=L_{i}=10-12 \mathrm{~cm}$ in Figure 9 also matches with what would be expected for an inertial range. The quoted lower limits of $l$ are dictated by spatial resolution, and the upper limits coincide with the forcing length scales of $L_{i} \sim 12$ and $10 \mathrm{~cm}$ in experiments 6 and 8, respectively. We note that values of $L_{i}$ are naturally close to the size of the Lissajoux curve executed by the cylinder while stirring, that is $L_{c}=2 A=15 \mathrm{~cm}$.

\section{Particle dispersion}

In the context of the inhomogeneous and anisotropic turbulence just described, we now examine both single-particle dispersion and particle-pair dispersion, also known as relative dispersion. Although the dispersion of particles must be three-dimensional, the flow has been shown to be strongly 
anisotropic (and increasingly so as the stirring rate increases). So, the aim here is to check if large-sized particle dispersion in strongly inhomogeneous and anisotropic turbulence satisfies the same scaling laws as tracers in homogeneous and isotropic 3D turbulence.

\subsection{Single-particle dispersion}

Single-particle dispersion can be be investigated by analysing the trajectory of a single particle, by calculating $\left\langle|\boldsymbol{\Delta}(\tau)|^{2}\right\rangle$, where $\boldsymbol{\Delta}(\tau)=\boldsymbol{X}(t+\tau)-$ $\boldsymbol{X}(t)$, where $\boldsymbol{X}(t)$ is the position of a particle at each time $t$ along its trajectory and $\tau$ is the time lag. Figure 10a shows $\left\langle|\boldsymbol{\Delta}(\tau)|^{2}\right\rangle / L i^{2}$ as a function of $\tau / \tau_{e}$ for each of the four particles in experiment 7. When $\tau / \tau_{e} \lesssim 0.25$, the ballistic dispersion regime holds, i.e. $\left\langle|\boldsymbol{\Delta}(\tau)|^{2}\right\rangle \propto \tau^{2}$, whereas when $\tau / \tau_{e} \gtrsim 0.6-0.7$, the mean-square displacement follows a diffusive regime $\left(\left\langle|\boldsymbol{\Delta}(\tau)|^{2}\right\rangle \propto \tau\right)$ over a brief time interval of length approximately equal to $\tau_{e}$, as shown in Figure 10c. The start of the diffusive regime coincides with the time required for the decay of the Lagrangian velocity auto-correlation of the particles $\left\langle U_{i}(t) U_{i}(t+\tau)\right\rangle /\left\langle U_{i}(t)^{2}\right\rangle$, that is $T_{L} / \tau_{e}=0.6$, as shown in Figure 10b. At $\tau / \tau_{e} \approx 2.5$, the mean-square displacement reaches a plateau. The brevity of the diffusive regime is due to the finite dimensions of the domain, which limit the particle's excursion at large times. Similar dimensionless curves of $\left\langle|\boldsymbol{\Delta}(\tau)|^{2}\right\rangle / L i^{2}$ and $\left\langle U_{i}(t) U_{i}(t+\tau)\right\rangle /\left\langle U_{i}(t)^{2}\right\rangle$ as a function of $\tau / \tau_{e}$ were displayed for all other experiments (not shown).

The dispersion regimes of Figure 10 may be also interpreted in terms of space instead of time. Thus $\tau / \tau_{e} \sim 0.25$ corresponds to the mean-square particle displacement $\left\langle|\boldsymbol{\Delta}(\tau)|^{2}\right\rangle / L_{i}^{2} \sim 0.16$, which corresponds to a root-meansquare displacement $L_{b} / L_{i}=\left(\left\langle|\boldsymbol{\Delta}(\tau)|^{2}\right\rangle\right)^{1 / 2} / L_{i} \sim 0.4$ or in dimensional terms 
a)

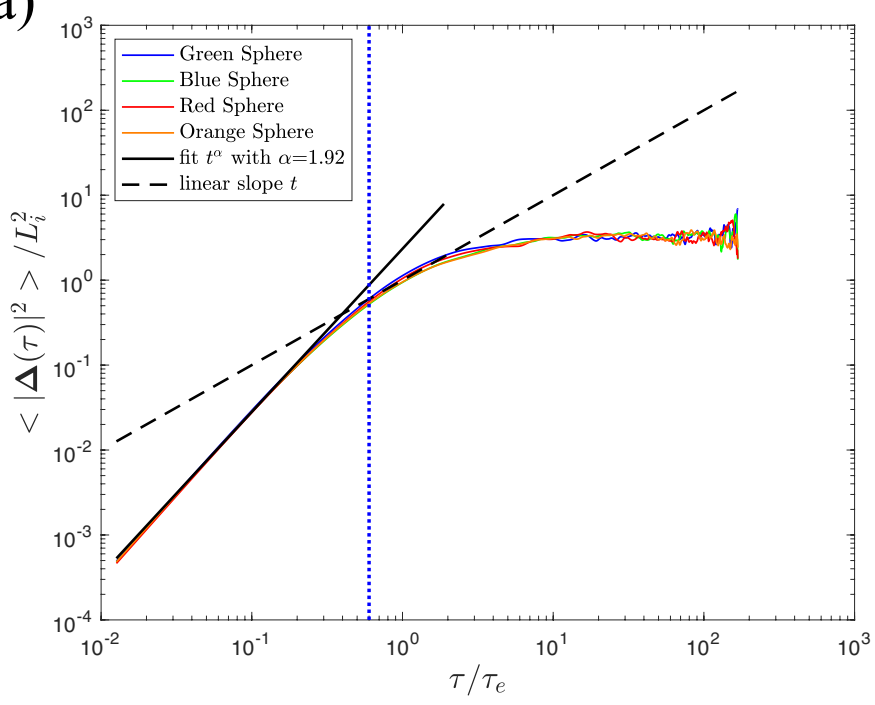

b)

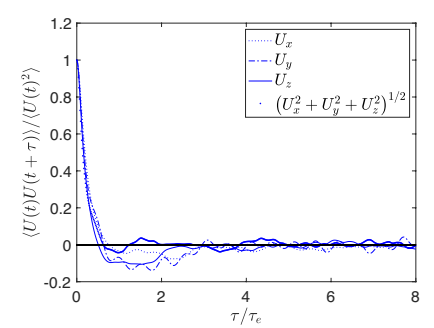

c)

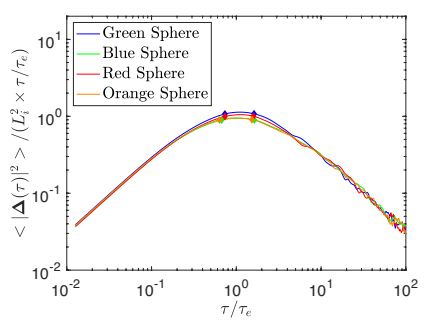

Figure 10: a) Mean square displacement relative to initial position as a function of time $\tau / \tau_{e}$ along the trajectories of the 4 particles in experiment 7 . The four particles have a similar behaviour. The black solid line represents a linear fit of the data (in logarithmic scales) up to $\tau / \tau_{e}=0.25$, which gives a slope of 1.92 , very close to the predicted slope of 2. The black dashed line represents a slope of 1 , indicating a diffusive regime. b) Lagrangian velocity auto-correlation function. The Lagrangian velocity auto-correlation time $T_{L} / \tau_{e} \sim 0.6$ is shown by the vertical blue dotted line in a). c) Single-dispersion compensated for the diffusive regime. The diamonds delimit the intervals over which $<|\boldsymbol{\Delta}(\tau)|^{2}>/\left(L_{i}^{2} \times \tau / \tau_{e}\right)$ is equal to its maximum within a tolerance of $5 \%$. This criterion is used to ascertain the presence of plateaus and hence the diffusive regime. The average width of such intervals for the four particles is $\Delta \tau / \tau_{e}=0.9$. Here the diffusive regime is observed between $T_{L} / \tau_{e} \lesssim \tau / \tau_{e} \lesssim 2.5 T_{L} / \tau_{e}$.

in experiment $7, L_{b}=\left(\left\langle|\boldsymbol{\Delta}(\tau)|^{2}\right\rangle\right)^{1 / 2} \sim 4.5 \mathrm{~cm}$. Hence, in this latter experiment, the ballistic regime remains valid for particle displacements below $\sim$ $5 \mathrm{~cm}$. On the other hand, $\tau / \tau_{e}=0.6$ corresponds to $\left\langle|\boldsymbol{\Delta}(\tau)|^{2}\right\rangle / L_{i}^{2} \sim 0.63$, or $L_{d} / L_{i}=\left(\left\langle|\boldsymbol{\Delta}(\tau)|^{2}\right\rangle\right)^{1 / 2} / L_{i} \sim 0.8$, or equivalently, in experiment $7, L_{d}=$ 
$\left(\left\langle|\boldsymbol{\Delta}(\tau)|^{2}\right\rangle\right)^{1 / 2} \sim 9 \mathrm{~cm}$, which means that the diffusive regime will apply to particle displacements larger than $9 \mathrm{~cm}$. Finally, the plateau reached by the dispersion curves ends when $\left\langle|\boldsymbol{\Delta}(\tau)|^{2}\right\rangle / L_{i}^{2} \sim 1.8$, or $\left(\left\langle|\boldsymbol{\Delta}(\tau)|^{2}\right\rangle\right)^{1 / 2} / L_{i} \sim 1.34$, or equivalently, in experiment $7,\left(\left\langle|\boldsymbol{\Delta}(\tau)|^{2}\right\rangle\right)^{1 / 2} \sim 15 \mathrm{~cm}$, which means that once the particles approach displacements around $15 \mathrm{~cm}$ the displacement is unable to on average increase further due to the limited dimensions of the domain.

Aside from showing the existence of the ballistic and diffusive regimes, the results of this analysis of single-particle dispersion are the above definition of $T_{L}$ and the characteristic dimensionless lengths $L_{b}$ and $L_{d}$ delimiting the different dispersion regimes, which will be used in the next section in the interpretation of particle-pair dispersion.

\subsection{Particle-pair dispersion}

To analyse particle-pair dispersion, we used both the traditional way of looking at the relative dispersion as a function of time and the fixed lengthscale method (FSLE). We will show in this section that the two methods are complementary. We first looked at the time evolution of the separation between particles $i$ and $j,\left|\boldsymbol{S}_{i j}(t)\right|=\left|\boldsymbol{X}_{i}(t)-\boldsymbol{X}_{j}(t)\right|$, by calculating the meansquare relative distance $\left\langle\left(\left|\boldsymbol{S}_{\boldsymbol{i j}}\right|-S_{0}\right)^{2}\right\rangle$ of pair $i j$ relative to the initial pair separation $\left|\boldsymbol{S}_{\boldsymbol{i j}}(0)\right|=S_{0}$. The values of $S_{0}$ were carefully chosen so that they span characteristic lengths of the system within the interval $S_{0}=[3,11] \mathrm{cm}$. This allowed a calculation of statistically representative ensemble averages of the mean-square relative distance. However, the sampling was uneven: the number of ensemble members was found to roughly linearly increase with intermediate values of $S_{0}$, be sometimes small at the lowest $(<3 \mathrm{~cm})$ and 
largest $(>30 \mathrm{~cm})$ values of $S_{0}$, and vary between pairs for the same $S_{0}$, especially for experiments with low stirring rates. For instance, in experiment 5 , the number of ensemble members averaged over the six pairs increased from 18 to 151 as $S_{0}$ increased from 3 to $11 \mathrm{~cm}$, and varied between pairs at $S_{0} \sim 3 \mathrm{~cm}$ from zero (pair 4) to 62 (pair 5), whereas in experiment 11, the number of ensemble members averaged over the six pairs increased from 17 to 172 for a similar set of values of $S_{0}$, and varied between pairs at $S_{0} \sim 3 \mathrm{~cm}$ from 8 (pair 5) to 28 (pair 6). The fact that sampling varies between particle pairs likely results from the fact that the turbulence is neither isotropic nor homogeneous.

In all experiments, as exemplified in Figure 11 for experiments 5 and 11, the mean-square relative distance $\left\langle\left(|\boldsymbol{S}|-S_{0}\right)^{2}\right\rangle$ shows three main trends: in the interval $\tau / \tau_{e}<0.25$, it evolves as $t^{2}$, clearly following a ballistic regime; when $0.25<\tau / \tau_{e}<0.6$, it varies as $t^{\beta}$ with variable $\beta$ values, $1.0<\beta<2.3$, so that there is no indication of a super-diffusive regime; when $\tau / \tau_{e} \gg T_{L} / \tau_{e}=0.6$, it finally grows more slowly (eventually becoming stationary) with short-period oscillations, reflecting the finite dimensions of the domain. The diffusive dispersion regime in the interval $\tau / \tau_{e}>0.6$ is equivocal, partly for the same reasons related to particle confinement as in single-particle dispersion, but also partly because the statistics are noisier.

The lack of identification of an intermediate super-diffusive regime can be explained by the narrow time windows $\left[t_{0}, T_{L}\right]$ that are available for this regime to exist, as shown in Table 3 for experiments 5 and 11 and the initial separations $S_{0}$ considered in Figure 11. For the time $t_{0}=\left(S_{0}^{2} / \varepsilon\right)^{1 / 3}$ marking the transition from the ballistic to the super-diffusive regime at a given ini- 

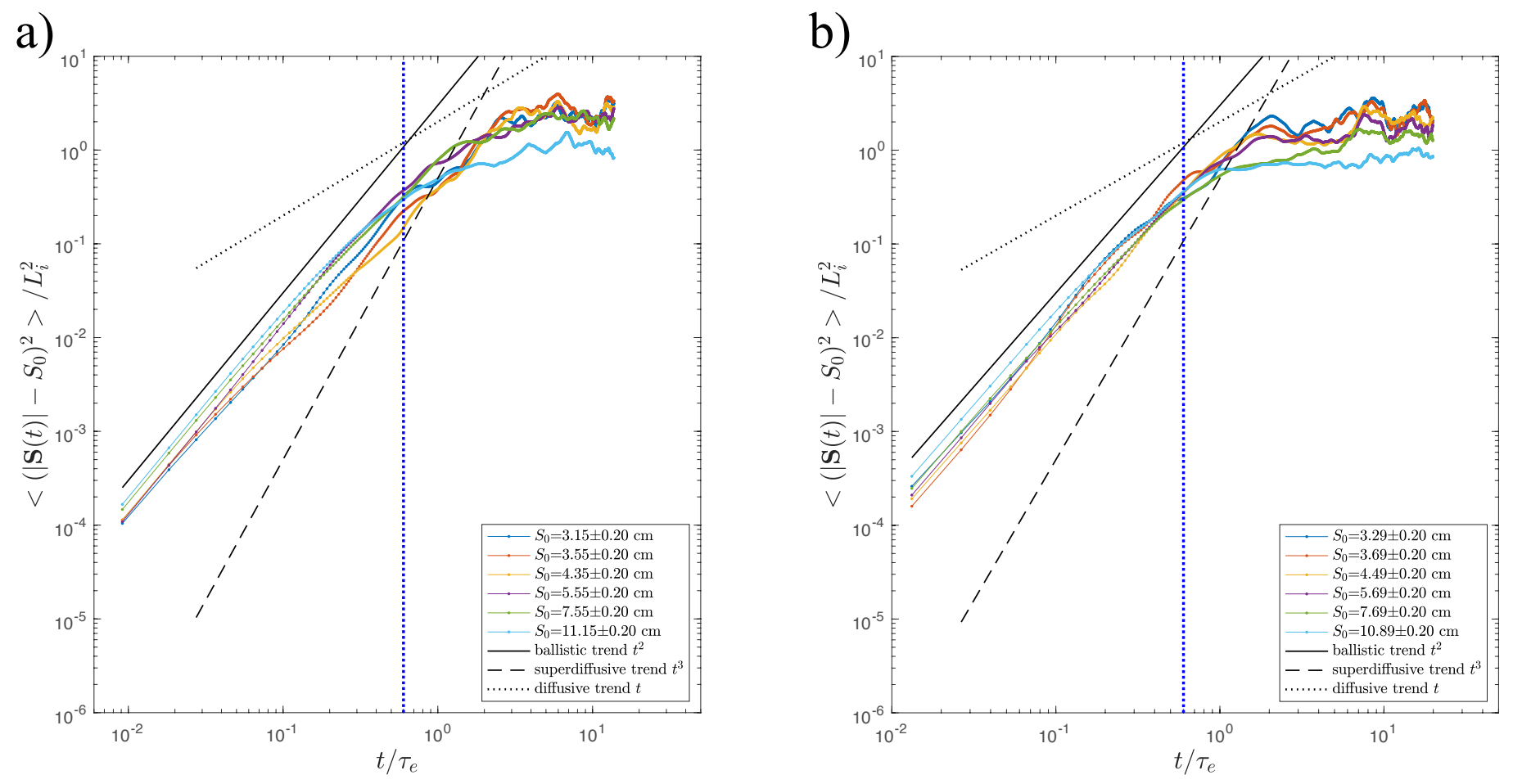

Figure 11: Mean square distance averaged over the six particle pairs for different initial separations $S_{0}$ as a function of time in a) experiment 5 and b) experiment 11 . The black solid, dashed and dotted lines represent slopes of 2, 3, and 1, respectively, characterizing the ballistic, super-diffusive and diffusive regimes. The time $T_{L} / \tau_{e}=0.6$ is shown by a vertical blue dotted line. 

$L_{b} \approx 4.5 \mathrm{~cm}$. expressed as:

\begin{tabular}{|ccc||cc|}
\hline \multicolumn{1}{|c|}{ Exp. 5 } & & Exp. 11 & \\
$S_{0}(\mathrm{~cm})$ & $t_{0} / T_{L}$ & $S_{0}(\mathrm{~cm})$ & $t_{0} / T_{L}$ \\
\hline 3.15 & 0.76 & 3.29 & 0.70 \\
3.55 & 0.83 & 3.69 & 0.75 \\
4.35 & 0.95 & 4.49 & 0.86 \\
5.55 & 1.11 & 5.60 & 1.00 \\
7.55 & 1.36 & 7.69 & 1.23 \\
11.15 & 1.77 & 10.89 & 1.55 \\
\hline
\end{tabular}

Table 3: Estimates of $t_{0} / T_{L}$ as a function of $S_{0}$ for experiments 5 and 11.

tial separation $S_{0}$ to be shorter and further separated from the Lagrangian integral time $T_{L}$ marking the start of the diffusive regime (see section 1 ), an increase in $\varepsilon$ and/or a smaller $S_{0}$ would be required. When $t_{0}>T_{L}$, the super-diffusive regime can not occur. The ratio of $t_{0}$ to $T_{L}$ can also be

$$
\frac{t_{0}}{T_{L}}=\frac{\left(S_{0}^{2} / \varepsilon\right)^{1 / 3}}{0.6 \tau_{e}}=\frac{\left(S_{0}^{2} / \varepsilon\right)^{1 / 3}}{0.6\left(L_{i}^{2} / \varepsilon\right)^{1 / 3}}=\frac{1}{0.6}\left(\frac{S_{0}}{L_{i}}\right)^{2 / 3} .
$$

So, $t_{0} / T_{L}>1$ is equivalent to $S_{0} / L_{i}>(0.6)^{3 / 2}=0.47$ or $S_{0}>4.7-5.6 \mathrm{~cm}$ (see Table 2), which is a limit that is very close to that found for the end of the ballistic regime in the single-particle dispersion, namely $L_{b} / L_{i} \sim 0.4$ or

52 As an alternative to the analysis of fixed-time average of inter-particle distances over the ensemble of particle pairs, we computed the Finite-Scale Lyapunov Exponent (FSLE). We thus calculated the function $\lambda(\delta)=\ln \rho /\langle\tau(\delta)\rangle$, 
where $\delta$ is the spatial separation between two particle trajectories and $\langle\tau(\delta)\rangle$ the mean time that $\delta$ takes to be amplified by a factor $\rho$. We took $\rho$ as equal to $\sqrt{2}$ (Corrado et al., 2017). We ultimately averaged $\lambda(\delta)$ over the six particle pairs. The results of the FSLE analysis are shown in Figure 12. In all experiments, two regimes $\left(\lambda(\delta) \propto \delta^{-\zeta}\right)$ are consistently found: the ballistic separation $(\zeta \sim 1)$ is present for $\delta<[0.77-0.99] L_{i}$; and the diffusive regime $(\zeta \sim 2)$ for $[0.77-0.99] L_{i}<\delta<[1.2-1.7] L_{i}$. We note that the length scale interval for diffusion is consistent with that found in the single particle analysis of the dispersion regime. For instance, in experiment 7, the diffusion regime is found for separations between $9 \mathrm{~cm}$ and $16 \mathrm{~cm}$, which agrees with the analysis of section 6.1. The fact that we find the transition to diffusive at $\delta \sim L_{i}$ shows that our estimates of the energy dissipation rate $\varepsilon$ derived from the particles, and consequently of a number of scales derived from $\varepsilon$, such as $L_{i}$, are reliable. Finally, it is to be noted that, whereas the traditional approach shows the ballistic regime with much less noise, the FSLE analysis shows the diffusive regime much more clearly. 

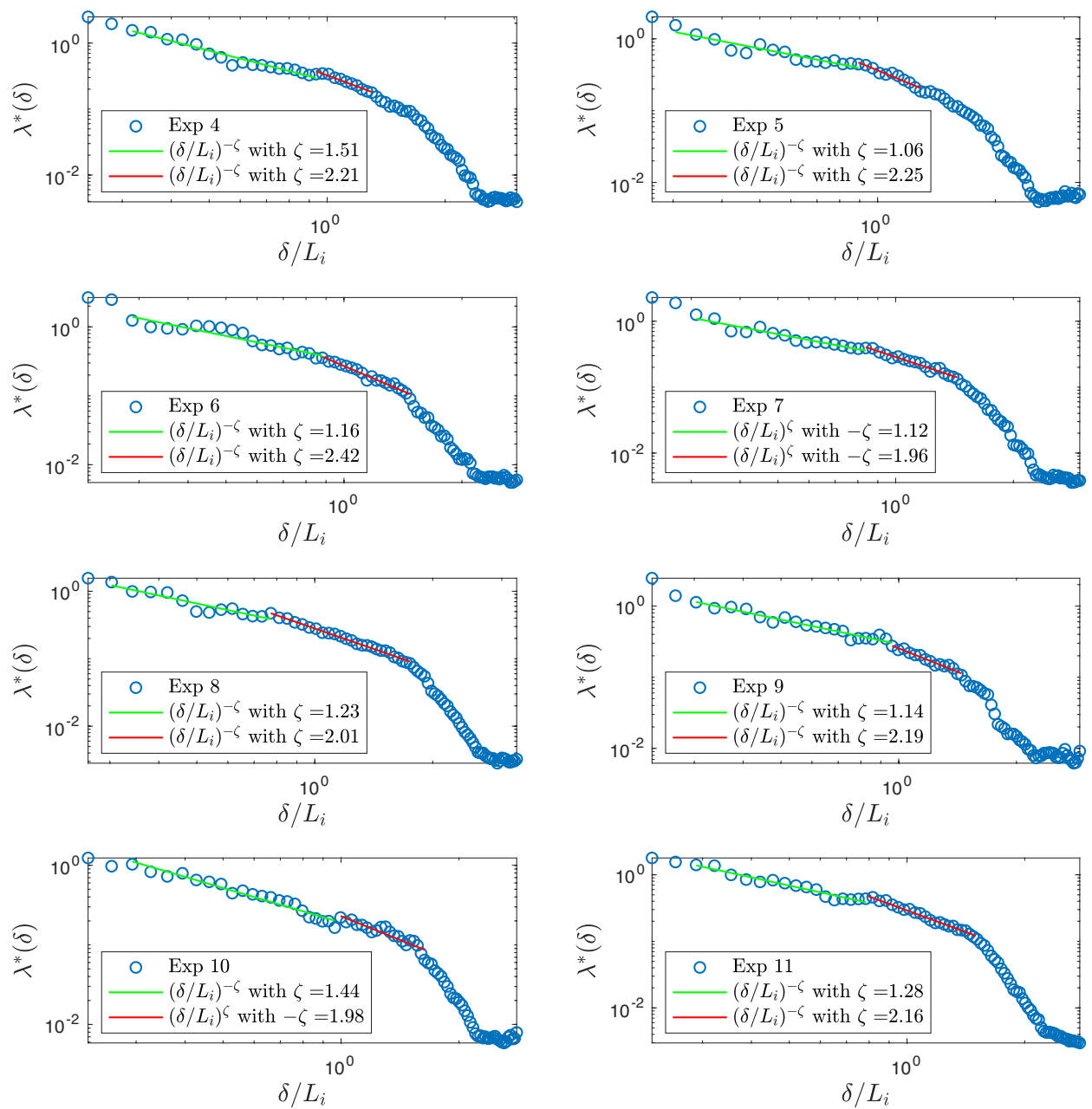

Figure 12: Lagrangian FSLE $\lambda(\delta)=\ln \rho /\left(\langle\tau(\delta)\rangle / \tau_{e}\right)$ as a function of $\delta / L_{i}$ for all experiments. The FSLE scaling exponent $\delta^{-\zeta}$ corresponds to: ballistic separation $(\zeta=1)$, and diffusive regime $(\zeta=2)$. 
Whereas two dispersion regimes were identified when the statistics were averaged over the six particle pairs, the dispersion between single pairs had more variability, especially at low stirring rate, as shown in Figure 13. For instance, in experiment 5, pair 2 separated as $t^{2.9}$ in the interval $0.13<$ $\tau / \tau_{e}<0.6$, whereas pair 6 separated as $t^{1.9}$ in the interval $0.01<\tau / \tau_{e}<0.6$. Similarly, in experiment 11, pair 3 separated as $t^{3.05}$ in the interval $0.2<$ $\tau / \tau_{e}<0.6$, whereas pair 2 separated as $t^{1.9}$ in the interval $0.01<\tau / \tau_{e}<$ 0.6. Individually, particle pairs could thus seemingly exhibit a super-diffusive behaviour extended outside of the expected time window $t_{0}<\tau<T_{L}$ (but overlapping with it). This variability affecting different dispersion pairs can be related to the variability of the Lagrangian correlation time of velocity differences (relative velocity between two particles of a pair) between the pairs separated by $S_{0} \sim 3 \mathrm{~cm}$, as shown in Figure 14. For instance, in experiment 5 , the velocity difference of pair 2 loses its memory of the initial separation at $\tau / \tau_{e}=0.13$, five times more rapidly than for pair 6 . This indicates not only that the ballistic regime ended earlier for pair 2 than for pair 6 but also that it ended earlier than the theoretical time $t_{0} / \tau_{e}=$ $t_{0} / T_{L} \times T_{L} / \tau_{e}=0.76 \times 0.6=0.46$. In practice, this corresponds to a better separation between $t_{0}$ and $T_{L}$, possibly allowing the Richardson regime to exist in this case. Similarly, in experiment 11, the velocity difference of pair 3 decorrelated at $\tau / \tau_{e}=0.19$ instead of $t_{0} / \tau_{e}=0.42$ and earlier by a factor of 3 than for pair 2. It is tempting to attribute the difference in the decorrelation time of the velocity difference between pairs to the inhomogeneity of the turbulence, although it is rather intricate to identify why it would affect the pairs differently. Nevertheless, for $S_{0} \sim 3 \mathrm{~cm}$, histograms of the particle 
a)

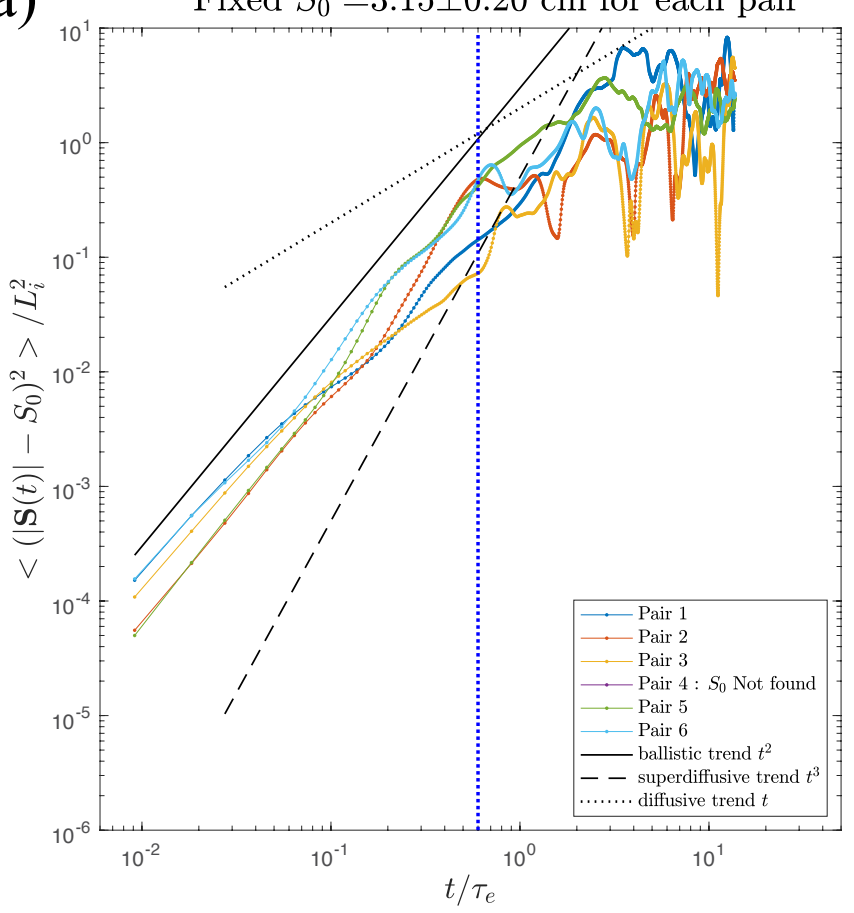

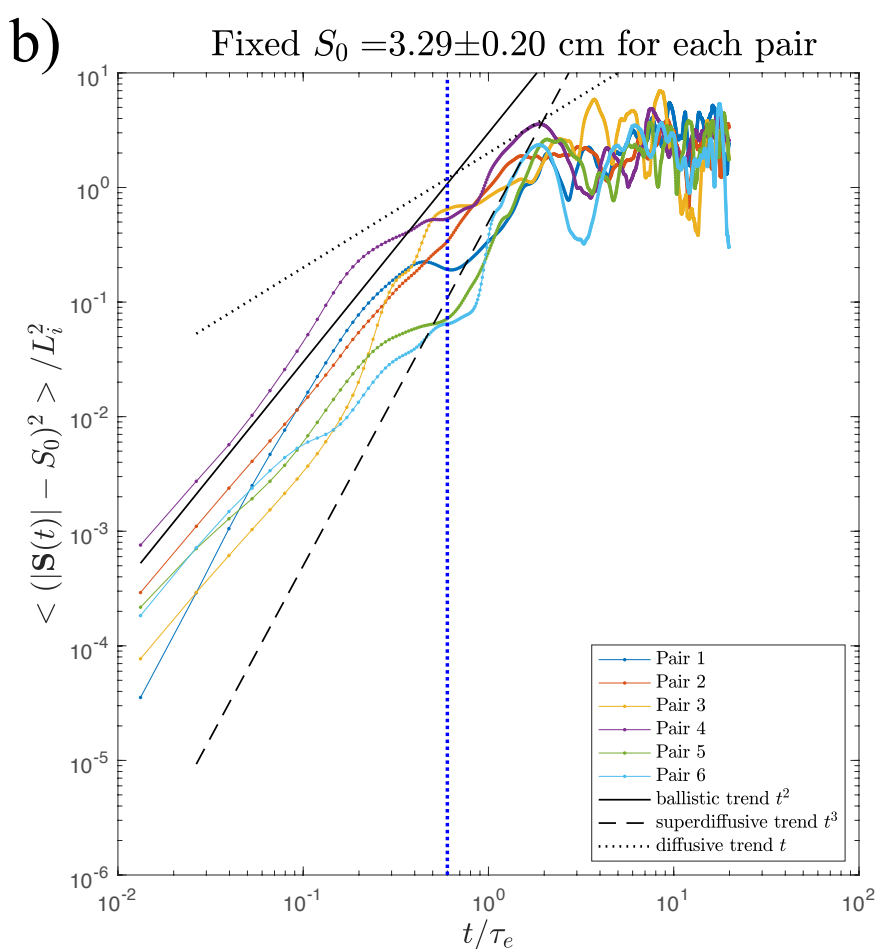

Figure 13: Mean square distance for each particle pair for $S_{0} \sim 3 \mathrm{~cm}$ as a function of time in experiments a) 5 and b) 11. The black solid, dashed and dotted lines represent slopes of 2, 3, and 1, respectively, characterizing the ballistic, super-diffusive and diffusive regimes. The time $T_{L} / \tau_{e}=0.6$ is shown by a vertical blue dotted line. The separation of the particle pair 4 was never less than $5.5 \mathrm{~cm} \mathrm{n}$ experiment 5 , and so that pair is not shown in a).

positions for each characteristic pair 2 and 6 , and 2 and 3 , respectively, in experiments 5 and 11, shown in Figure 15, reveal that the particle pairs whose velocity differences were decorrelating slowly were actually close to the bottom wall or the free surface, i.e. they were located in two highly inhomogeneous regions. 

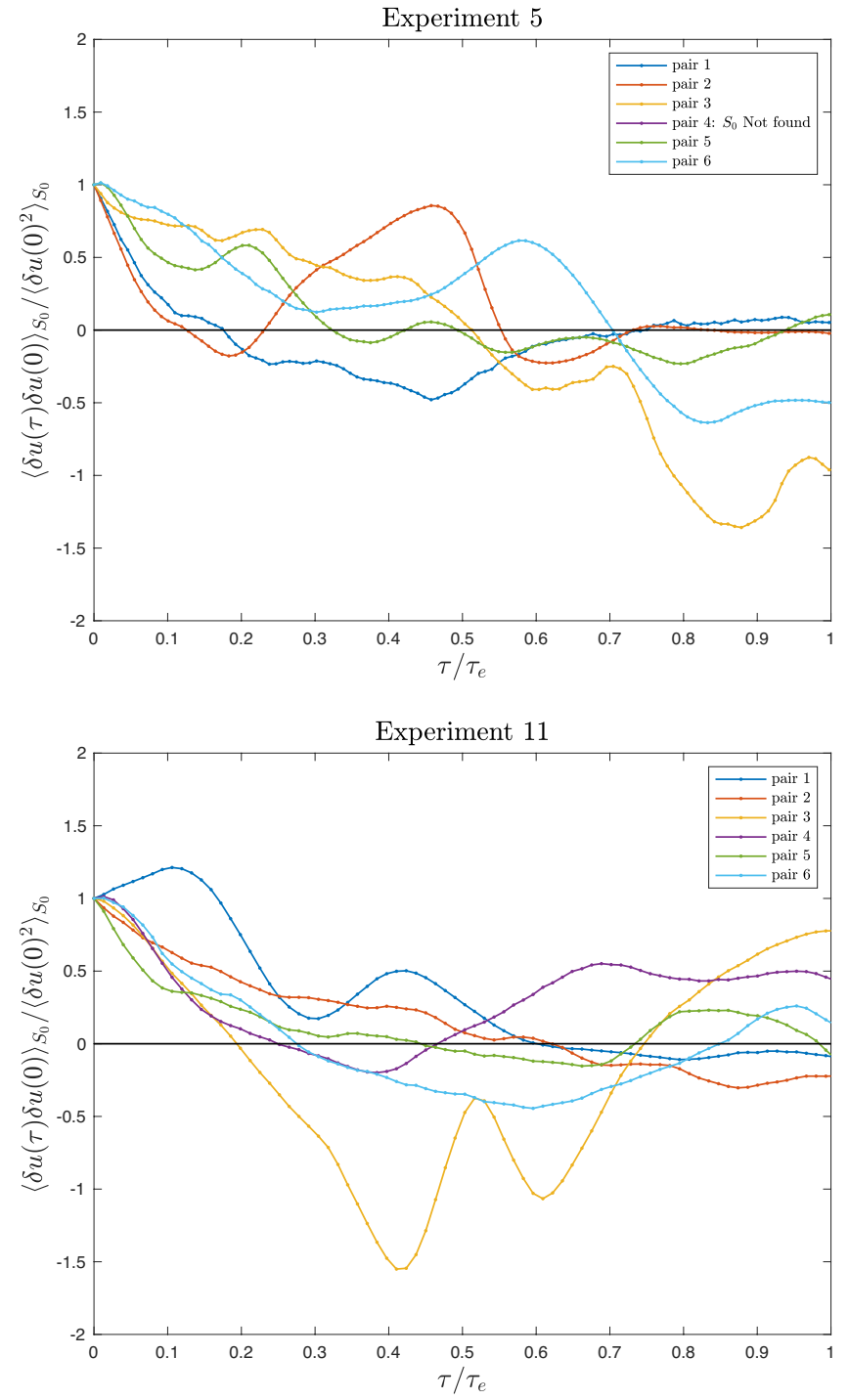

Figure 14: Lagrangian correlation of velocity differences between the particle pairs separated by $S_{0},\langle\delta u(\tau) \delta u(0)\rangle_{S_{0}} /\left\langle\delta u(0)^{2}\right\rangle_{S_{0}}$, as a function of $\tau / \tau_{e}$ for experiment $5\left(S_{0}=3.15\right.$ $\mathrm{cm})$ and experiment $11\left(S_{0}=3.29 \mathrm{~cm}\right)$. 


\section{a) Experiment 5}
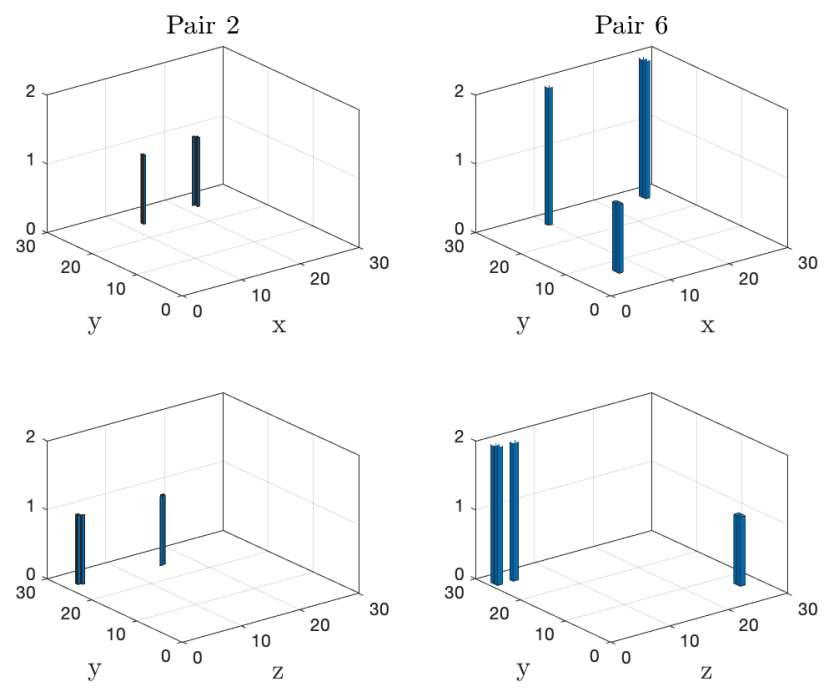

\section{b) Experiment 11}
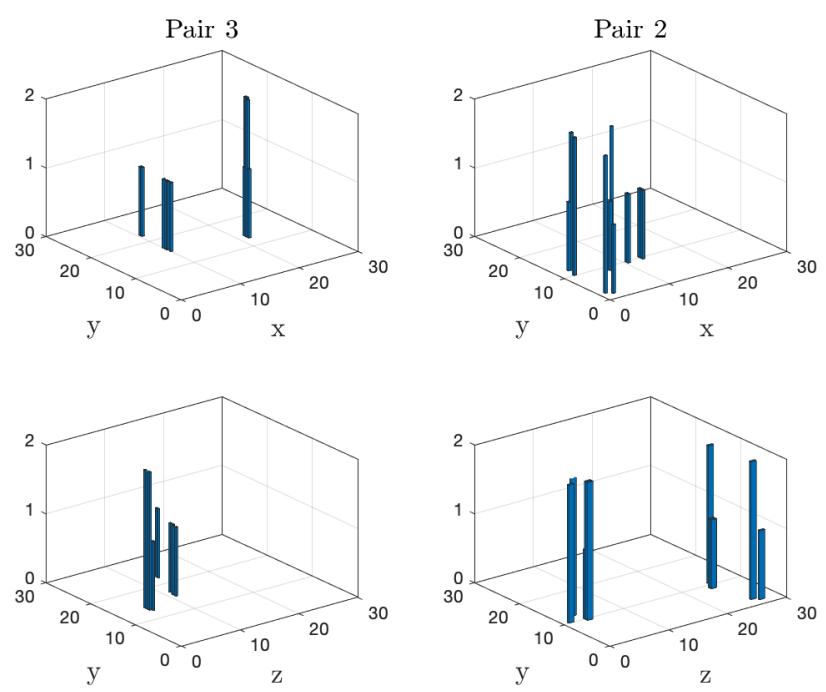

Figure 15: Histograms of the positions $X, Y, Z$ for the particle pairs 2 and 6 , and 2 and 3 in a) experiment 5 and b) experiment 11 when $S_{0} \sim 3 \mathrm{~cm}$. Each bar represents the number of times the $x, y$ or $z$ particle positions of a pair were encountered when the particle-pair distance was about $3 \mathrm{~cm}$ (which corresponds to the initial times contributing to the Lagrangian correlation of velocity differences between the particle pairs in Figure 14). 


\section{Conclusions}

In this experimental study, we departed from the theoretical framework for homogeneous and isotropic turbulence and the dispersion of fluid (tracer) particles to assess to what extent classic theories remain valid for the dispersion of large particles in inhomogeneous and anisotropic turbulence.

Our original experimental design consisted of stirring a fluid together with a few almost neutrally-buoyant finite-size particles contained in a rectangular tank including a mixed type of boundaries (no-slip and free surface). The stirring of the two phases (fluid/particle) was achieved by a cylinder executing a two-dimensional periodic Lissajoux figure enclosing a quarter of the volume of the tank. Our approach consisted of recording the dynamics of the particles in the entire volume of the tank, without using tracers. In doing so, we did not directly probe the turbulence over the entire inertial range, but over a limited scale range, which, in terms of length scales, extended from the particle size to the tank's dimensions. However, the velocities of the finitesize particles allowed us to determine the velocity-based properties of the turbulence with tolerable accuracy.

Despite our initial expectations of particle collisions, only particle-cylinder collisions had multiple occurrences, but their effect on the particle motion remained limited. This can partly be explained by the fact that when particles are in the proximity of the cylinder, they frequently are engulfed in the vortex surrounding the cylinder, which makes them flow around the cylinder instead of colliding with it.

The dynamics of the particles was clearly indicative of anisotropy and inhomogeneity of the turbulence at the particle scale. The walls and free- 
surface contributed to the inhomogeneity, as shown by the velocity field. The absence of forcing motion in the direction along the axis of the cylinder resulted in a preferred velocity direction of the particles normal to the cylinder axis. Consequently, random preferential locations and trapping of particles along the $x$ direction were recorded, especially at low stirring rates. Velocity fluctuations at the scale of the particles in this direction seemed insufficient to eject particles from their trapping regions. For a given period of the cylinder motion, the two opposite directions of stirring did not produce substantially different anisotropy, but produced a different inhomogeneity of the particle velocity fields.

Single-particle dispersion exhibited a ballistic regime at times shorter than the particle Lagrangian velocity auto-correlation time, and a short diffusive regime at longer times, in agreement with theoretical predictions for tracers in isotropic and homogeneous turbulence.

Particle-pair dispersion mostly agreed with the classic predictions for dispersion in 3D turbulence when averaged over the six pairs, as ballistic and diffusive regimes were found. The super-diffusive regime was not observed because the time $t_{0}$ was not sufficiently smaller than the Lagrangian correlation time $T_{L}$. So, a temporal window for super-diffusion did not exist, and the ballistic regime transitioned directly to the diffusive regime. However, individually, some particle pairs briefly gave indications of a super-diffusive regime following the Richardson law. These particle pairs were found to be characterised by a more rapid decorrelation of their velocity differences compared to other pairs. We further made a link between the variability in the timescale of transition from the ballistic regime to Richardson's law and 
the inhomogeneity of turbulence, by noting that larger decorrelation times tended to occur near the tank's boundaries. Overall, both single-particle and particle-pair dispersion mostly agree with the ballistic and diffusive behaviours expected for 3D dispersion in homogeneous and isotropic turbulence despite the inhomogeneity and anisotropy of the turbulence in our experiments.

\section{Acknowledgments}

The experiments were carried out in the Geodynamic Modelling Laboratory of the School of Earth, Atmosphere and Environment at Monash University. Authors J.J. Monaghan and C.A. Mériaux acknowledge support from former ARC Discovery grant DP 130104356 (Analysis and application of a Lagrangian turbulence model for smoothed particle hydrodynamics), and project with CSIRO Data61 of the Commonwealth Scientific and Industrial Research Organisation, "Measurement and Simulation of particle motion in forced turbulent flow". Author C.A. Mériaux thanks Brett A. Williams, Michael Ladd and Antonio Benci from the Monash Instrumentation Facility for their effective contribution to the experimental setup, Dr. Jisheng Zhao from the Department of Mechanical and Aerospace Engineering at Monash University for contributing to the experimental design, and Shantanu Bhat, Ph.D. candidate from the same department for his training on how to use the Twincat software. We thank the editor GertJan van Heijst and three anonymous referees for their comments, which contributed to the improvement of the manuscript. We acknowledge the suggestion of the FLSE analysis by one anonymous referee, to whom we are very grateful. 
Aurell, E., Boffetta, G., Crisanti, A., Paladin, G., Vulpiani, A., 1996. Growth of noninfinitesimal perturbations in turbulence. Physical review letters $77(7), 1262$.

Balachandar, S., Eaton, J. K., 2010. Turbulent dispersed multiphase flow. Annual review of fluid mechanics 42, 111-133.

Batchelor, G., 1950. The application of the similarity theory of turbulence to atmospheric diffusion. Quarterly Journal of the Royal Meteorological Society 76 (328), 133-146.

Bellani, G., Byron, M. L., Collignon, A. G., Meyer, C. R., Variano, E. A., 2012. Shape effects on turbulent modulation by large nearly neutrally buoyant particles. Journal of Fluid Mechanics 712, 41-60.

Biferale, L., Bodenschatz, E., Cencini, M., Lanotte, A. S., Ouellette, N. T., Toschi, F., Xu, H., 2008. Lagrangian structure functions in turbulence: A quantitative comparison between experiment and direct numerical simulation. Physics of Fluids 20 (6), 065103.

Bitane, R., Homann, H., Bec, J., 2012. Time scales of turbulent relative dispersion. Physical Review E 86 (4), 045302.

Boffetta, G., Cencini, M., Espa, S., Querzoli, G., 2000. Chaotic advection and relative dispersion in an experimental convective flow. Physics of Fluids 12 (12), 3160-3167.

Boffetta, G., Sokolov, I., 2002. Relative dispersion in fully developed turbulence: the richardson's law and intermittency corrections. Physical review letters 88 (9), 094501. 
Bourgoin, M., 2015. Turbulent pair dispersion as a ballistic cascade phenomenology. Journal of Fluid Mechanics 772, 678-704.

Bourgoin, M., Qureshi, N. M., Baudet, C., Cartellier, A., Gagne, C., 2011. Turbulent transport of finite sized material particles. In: Journal of Physics: Conference Series. Vol. 318. IOP Publishing, p. 012005.

Corrado, R., Lacorata, G., Palatella, L., Santoleri, R., Zambianchi, E., 2017. General characteristics of relative dispersion in the ocean. Scientific reports 7, 46291 .

Csanady, G. T., 1973. The fluctuation problem in turbulent diffusion. In: Turbulent Diffusion in the Environment. Springer, pp. 222-248.

Einstein, A., 1956. Investigations on the Theory of the Brownian Movement. Courier Corporation.

Fiabane, L., Zimmermann, R., Volk, R., Pinton, J.-F., Bourgoin, M., 2012. Clustering of finite-size particles in turbulence. Physical Review E 86 (3), 035301.

Gibert, M., Xu, H., Bodenschatz, E., 2010. Inertial effects on two-particle relative dispersion in turbulent flows. EPL (Europhysics Letters) 90 (6), 64005.

Gore, R., Crowe, C. T., 1989. Effect of particle size on modulating turbulent intensity. International Journal of Multiphase Flow 15 (2), 279-285.

Ibrahim, R. A., 2005. Liquid sloshing dynamics: theory and applications. Cambridge University Press. 
Klein, S., Gibert, M., Bérut, A., Bodenschatz, E., 2012. Simultaneous 3d measurement of the translation and rotation of finite-size particles and the flow field in a fully developed turbulent water flow. Measurement Science and Technology 24 (2), 024006.

Kolmogorov, A. N., 1941a. Energy dissipation in locally isotropic turbulence. In: Dokl. Akad. Nauk. SSSR. Vol. 32. pp. 19-21.

Kolmogorov, A. N., 1941b. The local structure of turbulence in incompressible viscous fluid for very large reynolds numbers. In: Dokl. Akad. Nauk SSSR. Vol. 30. pp. 299-303.

La Porta, A., Voth, G. A., Crawford, A. M., Alexander, J., Bodenschatz, E., 2001. Fluid particle accelerations in fully developed turbulence. Nature 409 (6823), 1017.

Machicoane, N., Volk, R., 2016. Lagrangian velocity and acceleration correlations of large inertial particles in a closed turbulent flow. Physics of Fluids 28 (3), 035113.

Monaghan, J., 2017. Sph-e simulation of 2d turbulence driven by a moving cylinder. European Journal of Mechanics-B/Fluids 65, 486-493.

Monaghan, J., Mériaux, C. A., 2018a. An sph study of driven turbulence near a free surface in a tank under gravity. European Journal of MechanicsB/Fluids 68, 201-210.

Monaghan, J., Mériaux, C. A., 2018b. What can we learn from large bodies moving in a turbulent fluid? European Journal of Mechanics-B/Fluids 72, $519-530$. 
Monin, Andrei, S., Yaglom, A. M., 2013. Statistical fluid mechanics, volume II: mechanics of turbulence. Vol. 2. Courier Corporation.

Mordant, N., Delour, J., Léveque, E., Michel, O., Arnéodo, A., Pinton, J.F., 2003. Lagrangian velocity fluctuations in fully developed turbulence: scaling, intermittency, and dynamics. Journal of Statistical Physics 113 (56), 701-717.

Mordant, N., Lévêque, E., Pinton, J.-F., 2004. Experimental and numerical study of the lagrangian dynamics of high reynolds turbulence. New Journal of Physics 6 (1), 116.

Ouellette, N. T., Xu, H., Bourgoin, M., Bodenschatz, E., 2006. Small-scale anisotropy in lagrangian turbulence. New Journal of Physics 8 (6), 102.

Qureshi, N. M., Bourgoin, M., Baudet, C., Cartellier, A., Gagne, Y., 2007. Turbulent transport of material particles: an experimental study of finite size effects. Physical review letters 99 (18), 184502.

Richardson, L. F., 1926. Atmospheric diffusion shown on a distanceneighbour graph. Proc. R. Soc. Lond. A 110 (756), 709-737.

Salazar, J. P., Collins, L. R., 2009. Two-particle dispersion in isotropic turbulent flows. Annual review of fluid mechanics 41, 405-432.

Sreenivasan, K. R., 1995. On the universality of the kolmogorov constant. Physics of Fluids 7 (11), 2778-2784.

Taylor, G. I., 1922. Diffusion by continuous movements. Proceedings of the london mathematical society 2 (1), 196-212. 
Teixeira, M., Belcher, S., 2000. Dissipation of shear-free turbulence near boundaries. Journal of Fluid Mechanics 422, 167-191.

Toschi, F., Bodenschatz, E., 2009. Lagrangian properties of particles in turbulence. Annual review of fluid mechanics 41, 375-404.

Valizadeh, A., Monaghan, J., 2015. Sph simulation of 2d turbulence driven by a cylindrical stirrer. European Journal of Mechanics-B/Fluids 51, 44-53.

Valizadeh, A., Monaghan, J. J., 2012. Smoothed particle hydrodynamics simulations of turbulence in fixed and rotating boxes in two dimensions with no-slip boundaries. Physics of Fluids 24 (3), 035107.

Vargaftik, N., Volkov, B., Voljak, L., 1983. International tables of the surface tension of water. Journal of Physical and Chemical Reference Data 12 (3), $817-820$.

Volk, R., Calzavarini, E., Leveque, E., Pinton, J.-F., 2011. Dynamics of inertial particles in a turbulent von kármán flow. Journal of Fluid Mechanics $668,223-235$.

Xia, H., Francois, N., Faber, B., Punzmann, H., Shats, M., 2019. Local anisotropy of laboratory two-dimensional turbulence affects pair dispersion. Physics of Fluids 31 (2), 025111.

Yeung, P., Pope, S., 1988. An algorithm for tracking fluid particles in numerical simulations of homogeneous turbulence. Journal of computational physics 79 (2), 373-416. 
Zandbergen, P., Dijkstra, D., 1987. Von kármán swirling flows. Annual review of fluid mechanics 19 (1), 465-491.

Zimmermann, R., Gasteuil, Y., Bourgoin, M., Volk, R., Pumir, A., Pinton, J.-F., for Turbulence, I. C., 2011. Tracking the dynamics of translation and absolute orientation of a sphere in a turbulent flow. Review of Scientific Instruments $82(3), 033906$.

\section{Appendix A. Particle lifting and transient times}

By lifting time we mean the time it takes for particles to lift off the bottom of the tank once the stirring motion has started. These lifting times inevitably reflect the time it takes for momentum to reach the bottom wall after flow initiation. Such a time is related to the time at which the cylinder reaches its minimum distance from the bottom wall $\left(y_{c}=A\right)$ during its first cycle, and thus should be proportional to $T$. For initial downward cylinder motion, the cylinder first reaches this height at a time $T / 8$, which potentially translates into an increase by a factor $3 / 1.75 \sim 1.7$ of the lifting times between the cases with $T=3 \mathrm{~s}$ and $T=1.75 \mathrm{~s}$. This appeared to be consistent with what we observed, as the ratio of the mean of all the lifting times at $T=3$ $\mathrm{s}$ to those at $T=1.75 \mathrm{~s}$ was 1.79 . For initial upward cylinder motion, the cylinder first reaches its lower height at a time $3 T / 8$. So, one would expect that particle lifting in this latter case takes three times longer than for the initial downward motion. We found an increase of that order, at $T=3 \mathrm{~s}$, as the ratio of the mean of all the lifting times for initial upward motion to those for initial downward motion was 2.25. In any case, this lifting time 

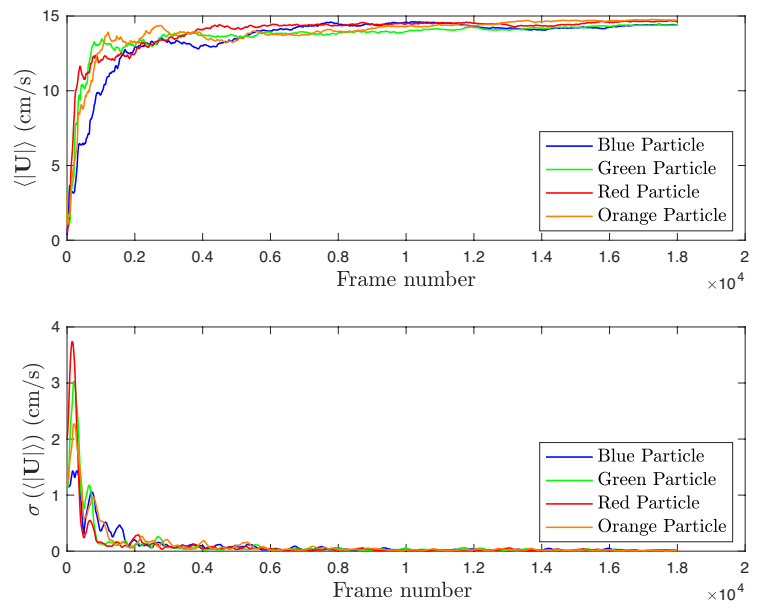

Figure A.16: (a) mean particle velocity $\langle|\boldsymbol{U}|\rangle$ as a function of the number of frames. (b) Standard deviation $\sigma(\langle|\boldsymbol{U}|\rangle)$ over neighbouring frames as a function of the number of frames. Data from experiment 11 have been used as an example.

can only be regarded as an indication of how long it takes for momentum to reach the bottom wall after flow initiation, which takes from one to five loops of the cylinder motion only.

To further assess how long it takes for the turbulent flow to be fully established in the tank, we calculated the mean velocity of the particles as a function of frame number. As shown in Figure A.16a, the mean velocity reaches a plateau, whose start signals the onset of stationary turbulence in the tank. The onset of the plateau is detected numerically by an algorithm which estimates from which frame the standard deviation over a number of neighbouring frames corresponding to one third of the cylinder period is first less than $5 \%$ (Figure A.16b). Times $t_{s}$ at which the transient is over in all experiments are given in the last column of Table 1. They range from 20 to 34 cycles of the cylinder (equivalent to 3071 to 4711 frames over a total of 
18,000 frames).

\section{Appendix B. Velocity time series, collisions and filtering}

The time series of the velocity are characterized by two types of peaks. Some are short-lived (from a fifth to half a second), and result from a particle colliding with the cylinder, and being kicked in either the $y$ or $z$ direction at a speed that can exceed the maximal speed of the cylinder. Those kicks often result in the particle subsequently rebounding against a wall. The transfer of momentum that takes place is all the more important as the speed of the cylinder is high. Table 1 of section 2.4 gives the total number of these collisions for each experiment over 100 cycles. On average, a particle has a collision with the cylinder every 4 to 7 cycles. Given the typical collision duration, 4 to $10 \%$ of the time series recorded thus are affected by particlecylinder collisions. The second type of peaks last approximately one to two seconds, and occur when particles happen to be in the wake of the cylinder. There are rare collisions with the cylinder rod, and we counted only two occurrences of collision between particles across 11 experiments (only 8 experiments have been used).

To filter the collisions from the measured velocities, we assume that the collisions only transfer momentum to the $y$ and $z$ particle velocity components. The collisions are identified in the time series of $U_{y}$ and $U_{z}$ by automatically finding peaks exceeding a velocity threshold that is adjusted using the recorded videos. The velocities are then smoothed based on a local regression using weighted linear least-squares and a second-degree polynomial model, assigning lower weight to outliers in the regression. A zero weight 

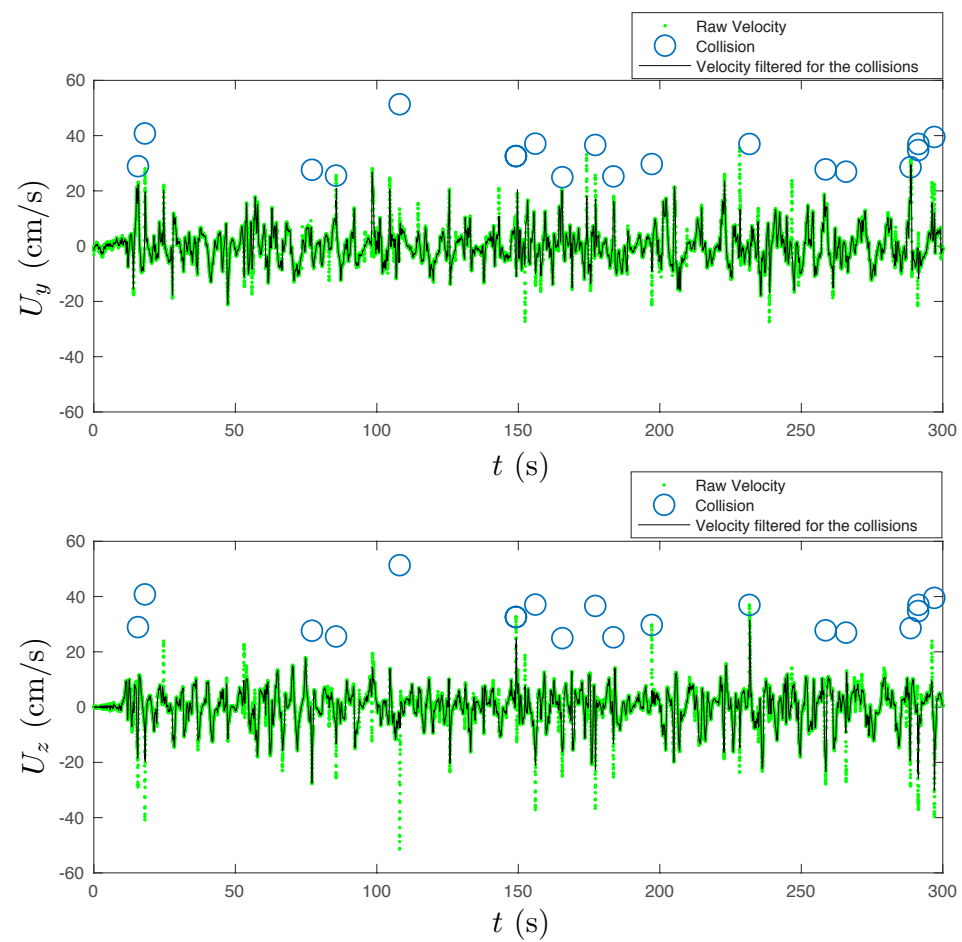

Figure B.17: Time series of the velocity components $U_{y}$ and $U_{z}$ for the green particle in experiment 6 before and after filtering the collisions.

is assigned to the data outside six mean absolute deviations. Figure B.17 shows an example of the procedure.

Figure B.18 shows the efficiency of the momentum transfer from the cylinder to the particles as well as the lack of impact from filtering. We note that the transients were not removed from the time series of the velocity in this instance. On average, the velocity of the particles is about 35\% that of the cylinder but the standard deviations are larger when the stirring is more vigorous (greater $U_{c}=\overline{u_{c}}$ ), which appears to result at least partly from particle collisions, as shown by the fact that the standard deviation is substantially 


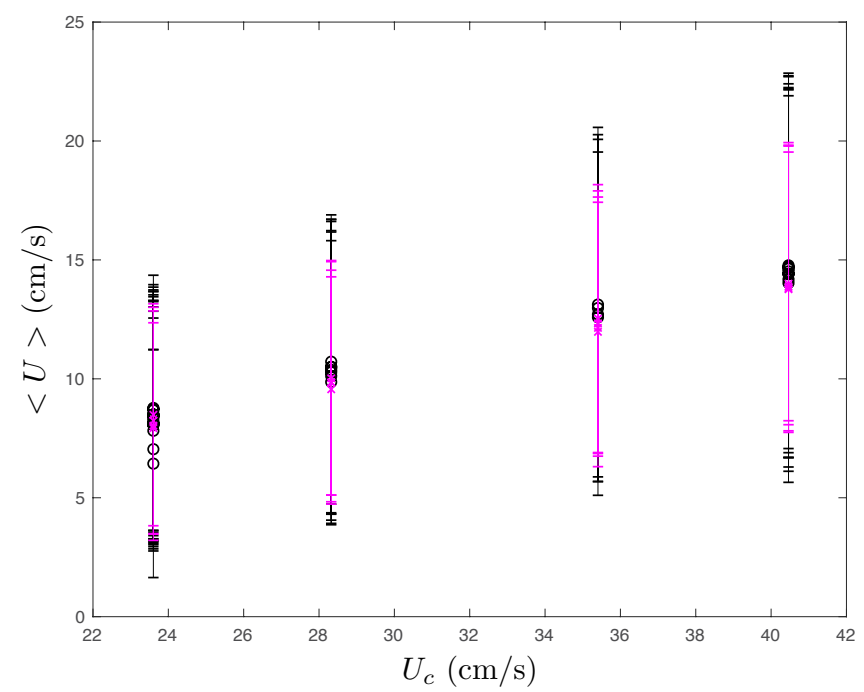

Figure B.18: Mean speed of the particles $\langle U\rangle=\langle|\boldsymbol{U}|\rangle$ including its standard deviation as a function of the mean cylinder velocity $U_{c}=\overline{u_{c}}$ for all experiments (in black). The mean and standard deviation from experiments 4, 6, 7 and 11 that were estimated after filtering the velocity for collisions with the cylinder are presented in magenta.

reduced when collisions are filtered (magenta lines).

We further assessed that the collisions have only minor impact on the velocity and acceleration distributions (not shown). 